AperTO - Archivio Istituzionale Open Access dell'Università di Torino

\title{
Multivariate factor-based processes with Sato margins
}

\section{This is the author's manuscript}

Original Citation:

Availability:

This version is available http://hdl.handle.net/2318/1691488

since 2019-02-09T18:49:53Z

Published version:

DOI:10.1142/S021902491850005X

Terms of use:

Open Access

Anyone can freely access the full text of works made available as "Open Access". Works made available under a Creative Commons license can be used according to the terms and conditions of said license. Use of all other works requires consent of the right holder (author or publisher) if not exempted from copyright protection by the applicable law. 


\title{
Multivariate factor-based processes with Sato margins
}

\author{
Marina Marena \\ Department of Economics and Statistics, \\ Università di Torino. \\ Andrea Romeo \\ Department of Economics and Statistics, \\ Università di Torino and Intesa Sanpaolo. \\ Patrizia Semeraro \\ Department of Mathematical Sciences G. Lagrange, \\ Politecnico di Torino. ${ }^{1}$
}

\footnotetext{
${ }^{1}$ Corresponding Author.
} 


\begin{abstract}
We introduce a class of multivariate factor-based processes with the dependence structure of Lévy $\rho \alpha$-models and Sato marginal distributions. We focus on variance gamma and normal inverse Gaussian marginal specifications for their analytical tractability and fit properties. We explore if Sato models, whose margins incorporate more realistic moments term structures, preserve the correlation flexibility in fitting option data. Since $\rho \alpha$-models incorporate nonlinear dependence, we also investigate the impact of Sato margins on nonlinear dependence and its evolution over time. Further, the relevance of nonlinear dependence in multivariate derivative pricing is examined.
\end{abstract}

Journal of Economic Literature Classification: G12, G13

Keywords: Finance, multivariate asset models, Lévy processes, Sato processes, nonlinear dependence. 


\section{Introduction}

A number of Lévy processes, which extend the classical Black-Scholes benchmark, has been adopted to represent single-asset returns and have been extended in various ways to represent returns on several assets. These processes are in law subordinated Brownian motions, where the subordinator represents a stochastic change of time. The first subordinated multivariate model was constructed by considering a common time change to all assets represented by a univariate subordinator (see Madan and Seneta (1990) and Luciano and Schoutens (2006)). Unfortunately, the resulting models exhibited several shortcomings including the lack of independence between asset returns and a limited span of linear correlations. Even though both empirical evidence existed and theoretical tools were available, time changed Lévy processes with multivariate subordinators appeared only recently. For instance, Eberlein and Madan (2010) considered independent changes of time, while Semeraro (2008) and Luciano and Semeraro (2010) constructed a multivariate subordinator composed of a common component and an idiosyncratic component, named factor based subordinator. By so doing, they introduced the $\rho \alpha$-Lévy processes, which represent log-returns as the sum of an idiosyncratic and a systematic component. While the stock-specific factors are independent, the common factors, reacting to the market general level of activity, are dependent. The model is economically intuitive, while preserving analytical tractability. Furthermore, under suitable conditions, they retain marginal distributions to be variance gamma and normal inverse Gaussian processes. The construction in Luciano and Semeraro (2010) extends the $\alpha$ variance gamma dependence structure in Semeraro (2008), improving the correlation flexibility, by using correlated Brownian motions.

The correlation flexibility of these models has been studied in the historical setting by Luciano et al. (2016) and in the risk neutral one by Marena et al. (2015). In both cases they provide an overall good performance. Nevertheless, Lévy models have some drawbacks in option pricing applications at the marginal level. It is well known that the Lévy models are not capable of fitting both strike and time to maturity dimensions of the volatility surface. To get around this shortcoming, Carr et al. (2007) proposed the use of Sato processes, having a more realistic moment term structure, and Guillaume (2012) has shown that the Sato extension of the $\alpha$ variance gamma introduced by Semeraro (2008) enhances marginal fit both for low-volatility and high-volatility regime periods, while preserving the correlation structure. These features are confirmed by Boen and Guillaume (2016) who introduce a multivariate extension of the difference of Gamma processes by Finlay and Seneta (2008), in both Lévy and Sato versions.

Within this backdrop, building on Guillaume (2012) approach, we propose a class of models (called $\rho \alpha$-Sato models) able to incorporate the dependence structure of the $\rho \alpha$-models and the marginal properties of Sato processes. Indeed, the $\rho \alpha$-models are able to achieve higher correlations compared with $\alpha$-models. Specifically, we build a process with Sato margins on $\rho \alpha$-models with self-decomposable time one marginal dis- 
tributions. We introduce the class of $\rho \alpha$-Sato processes, although a complete analysis of the properties of the general process is out of the aim of the present paper. We focus on variance gamma and normal inverse Gaussian marginal distributions, for their analytical tractability and fit properties in the risk neutral setting. We empirically analyse their fit on option data. In particular, we explore if Sato models, whose margins are known to improve the marginal fit, preserve the correlation flexibility. These classes of models have two different sets of parameters: idiosyncratic parameters, i.e., stock-specific, and common ones. Marginal distributions do not depend on common parameters, which are usually used to fit the correlation structure, see, e.g., Luciano and Semeraro (2010), Guillaume (2012) and Luciano et al. (2016). This structure allows us to perform a satisfactory calibration of the marginal processes and, for given pairs of marginal parameters, to compare the correlation range allowed by the $\rho \alpha$-Sato process with the correlation range allowed by their Lévy counterpart. We then employ a joint calibration procedure, by setting a tollerance level for correlation errors, to assess the overall goodness of fit of the class of models. Further, the $\rho \alpha$-dependence structure incorporates nonlinear dependence, as shown in Luciano and Semeraro (2010). While correlation is time independent in both models, comoments are time independent in the $\rho \alpha$-Sato models and time-dependent in $\rho \alpha$-models. We therefore evaluate the impact of Sato margins on nonlinear dependence and its evolution over time. Since we consider couples of assets, two common parameters drive the dependence structure. Thus, we can move nonlinear dependence leaving the correlation level fixed. We conclude our analysis by measuring the impact of nonlinear dependence on two-assets derivative pricing, under $\rho \alpha$-Sato and $\rho \alpha$-models.

The paper is organized as follows. Section 1 recalls the factor-based Lévy models, whose specifications are described in the Appendix. Section 2 introduces the factorbased model with Sato marginal processes, which we call factor-based Sato process. Their variance gamma and normal inverse Gaussian specifications are introduced in Section 3. Models calibrations and correlation flexibility analysis are performed in Section 4. Section 5 examines the impact of linear and nonlinear dependence on multiasset derivative prices. Finally, Section 6 concludes.

\section{Factor-based subordinated Lévy processes}

This section recalls the $\rho \alpha$-models introduced in Luciano and Semeraro (2010). Their main properties and their specifications with variance gamma (VG) and normal inverse Gaussian (NIG) marginal processes are provided in Appendix A.

The $\rho \alpha$-models are factor-based subordinated Brownian motions constructed as the sum of two independent subordinated Brownian motions. The first has independent components, while the second is a Brownian motion with correlated marginal processes which are subordinated by a common subordinator. 
Formally, let $\boldsymbol{B}$ be a $n$-dimensional Brownian motion with independent components and Lévy triplet $(\boldsymbol{\mu}, \Sigma, 0)$, where $\Sigma=\operatorname{diag}\left(\sigma_{1}^{2}, \ldots, \sigma_{n}^{2}\right)$ and $\boldsymbol{\mu}=\left(\mu_{1}, \ldots, \mu_{n}\right)$. Let $\boldsymbol{B}^{\rho}$ be a correlated $n$-dimensional Brownian motion, with correlations $\rho_{i j}$, marginal drifts $\boldsymbol{\mu}^{\rho}=\left(\mu_{1} \alpha_{1}, \ldots, \mu_{n} \alpha_{n}\right)$ and diffusion matrix $\left(\Sigma^{\rho}\right)_{i j}:=\left(\rho_{i j} \sigma_{i} \sigma_{j} \sqrt{\alpha_{i}} \sqrt{\alpha_{j}}\right)_{i j}$.

The $\mathbb{R}^{n}$-valued subordinated process $\boldsymbol{Y}=\{\boldsymbol{Y}(t), t>0\}$ defined by

$$
\boldsymbol{Y}(t)=\left(\begin{array}{c}
B_{1}\left(X_{1}(t)\right)+B_{1}^{\rho}(Z(t)) \\
\ldots \\
B_{n}\left(X_{n}(t)\right)+B_{n}^{\rho}(Z(t))
\end{array}\right),
$$

where $X_{j}$ and $Z$ are independent subordinators, independent from $\boldsymbol{B}$, and $\boldsymbol{B}^{\rho}$ is a factor-based subordinated Brownian motion. $\boldsymbol{Y}$ is also indicated as $\rho \alpha$-model.

Obviously, whenever all the parameters $\rho_{i j}$ collapse to 0 across different components, i.e. $\rho_{i j}=0$, for $i \neq j, \rho_{i j}=1$, for $i=j$, we have a version of the model in which Brownian motions are independent. This version has been introduced in Semeraro (2008) and is named $\alpha$-model.

We write $\boldsymbol{Y}:=\boldsymbol{Y}(1)$ to indicate the random vector whose distribution is the time one distribution of the process $\boldsymbol{Y}(t)$. We say that $\boldsymbol{Y}$ has a $\rho \alpha$-distribution.

Luciano and Semeraro (2010) (Theorem 5.1) proved that the marginal return $j$ is a Brownian motion with parameters $\mu_{j}$ and $\sigma_{j}$ subordinated by the $j$-th marginal process $G_{j}(t)$ of a factor based-subordinator $\boldsymbol{G}(t)$. A multidimensional factor-based subordinator $\{\boldsymbol{G}(t), t \geq 0\}$ is defined as follows

$$
\boldsymbol{G}(t)=\left(X_{1}(t)+\alpha_{1} Z(t), \ldots, X_{n}(t)+\alpha_{n} Z(t)\right), \quad \alpha_{j}>0, j=1, \ldots, n,
$$

where $\boldsymbol{X}(t)=\left\{\left(X_{1}(t), \ldots, X_{n}(t)\right), t \geq 0\right\}$ and $\{Z(t), t \geq 0\}$ are independent subordinators with zero drift, and $\boldsymbol{X}(t)$ has independent components. They represent the idiosyncratic and the common factors of trading activity. Indeed, the following equality in law holds

$$
Y_{j}(t) \stackrel{\mathcal{L}}{=} \mu_{j} G_{j}(t)+\sigma_{j} W\left(G_{j}(t),\right.
$$

where $\underline{\mathcal{L}}$ is equality in law. The marginal laws of $\boldsymbol{Y}(t)$ are therefore one-dimensional subordinated Brownian motions. We call factor-based distribution the time one distribution of a factor based subordinator, thus in this paper a factor based distribution is infinitely divisible. We call the factor-based distribution of the subordinator $\boldsymbol{G}$ the subordinating distribution of the corresponding $\rho \alpha$-model and we say that the $\rho \alpha$-distribution is associated to the factor-based distribution of $\boldsymbol{G}$.

\section{Factor-based Sato processes}

This section introduces a class of multivariate processes with Sato marginal processes constructed from the $\rho \alpha$-distributions. Sato processes are self-similar processes with 
independent increments, a.s. right-continuous with left limits. A stochastic process $X_{t}$ is a self-similar process if, for every $c>0$, there is a positive number $a_{c}$ such that $X(c t)=\mathcal{L}$ $a_{c} X(t)$. A Sato process can be constructed from self-decomposable distributions, which are a subclass of infinitely divisible distributions.

Definition 2.1 (Sato (1999), Definition 15.1). The distribution of a random variable $\boldsymbol{X}$ on $\mathbb{R}^{d}$ is self-decomposable (s.d.) or of class $L$ if $\forall b>1 \exists \boldsymbol{X}_{b}$ on $\mathbb{R}^{d}$ independent of $\boldsymbol{X}$ such that:

$$
\psi_{\boldsymbol{X}}(\mathbf{u})=\psi_{\boldsymbol{X}}\left(b^{-1} \mathbf{u}\right) \psi_{\boldsymbol{X}_{b}}(\mathbf{u}), \quad \mathbf{u} \in \mathbb{R}^{d},
$$

where $\psi_{\boldsymbol{X}}$ and $\psi_{\boldsymbol{X}_{b}}$ are the characteristc functions of $\boldsymbol{X}$ and $\boldsymbol{X}_{b}$, respectively.

The probability law of a Sato process at time $t$ is obtained by scaling the selfdecomposable law of $\boldsymbol{X}$ at unit time (see Carr et al. (2007)):

$$
\boldsymbol{X}(t) \stackrel{\mathcal{L}}{=} t^{h} \boldsymbol{X}
$$

where $h$ is the self-similar exponent. Sato processes are processes with independent but time inhomogeneous increments. Nevertheless the parameter $h$ is common to all components, thus inhomogeneity affects the sample path of each component in the same way.

Guillaume (2012) extended the $\alpha$-VG model introduced by Semeraro (2008) introducing Sato one dimensional marginal processes to enhance marginal fit both for lowvolatility and high-volatility regime periods. By so doing marginal distributions have different scale parameters and inhomogeneity of sample paths affects differently marginal processes. Using Guillaume (2012) approach we now define a multivariate stochastic process with Sato one dimensional processes, starting from $\rho \alpha$-distributions.

Firstly, we provide conditions for the factor-based distribution of the multivariate subordinator $\boldsymbol{G}$ to be self-decomposable, not only at the marginal level.

Proposition 2.1. If $\boldsymbol{G}$ has a factor-based distribution with self-decomposable components $X_{j}$ and $Z$, then $\boldsymbol{G}$ is self-decomposable.

Proof. $\boldsymbol{G}$ is self decomposable. Let $\psi_{\boldsymbol{G}}$ the characteristic function of $\boldsymbol{G}$. Since $X_{i}$ and $Z$ are s.d. for any $i=1, \ldots, n$ and for any $b>1$ it exists $X_{i}^{(b)}$ independent of $X_{i}$ and $Z^{(b)}$ independent of $X_{i}$ and $Z$ such that:

$$
\begin{aligned}
\psi_{\boldsymbol{G}}(\boldsymbol{u}) & =\prod_{j=1}^{n} \psi_{j}\left(u_{j}\right) \psi_{Z}\left(\sum_{j=1}^{n} \alpha_{j} u_{j}\right) \\
& =\prod_{j=1}^{n} \psi_{j}\left(b^{-1} u_{j}\right) \psi_{X_{j}^{(b)}}\left(u_{j}\right) \psi_{Z}\left(b^{-1} \sum_{j=1}^{n} \alpha_{j} u_{j}\right) \psi_{Z^{(b)}}\left(\sum_{j=1}^{n} \alpha_{j} u_{j}\right) \\
& =\psi_{\boldsymbol{G}}\left(b^{-1} \boldsymbol{u}\right) \prod_{j=1}^{n} \psi_{X_{j}^{(b)}}\left(u_{j}\right) \psi_{Z^{(b)}}\left(\sum_{j=1}^{n} \alpha_{j} u_{j}\right)=\psi_{\boldsymbol{G}}\left(b^{-1} \boldsymbol{u}\right) \psi_{\boldsymbol{G}^{(b)}}(\boldsymbol{u}) .
\end{aligned}
$$

Obviously $\boldsymbol{G}^{(b)}$ is independent of $\boldsymbol{G}$. 
In the following proposition we give conditions for the $\rho \alpha$-distributions to have one dimensional Sato marginal distributions.

Proposition 2.2. If $\boldsymbol{G}$ has a factor-based distribution with self-decomposable components $X_{j}$ and $Z$, then the associated $\rho \alpha$-distribution has self-decomposable marginal distributions.

Proof. Theorem 5.1 in Luciano and Semeraro (2010) proved that $Y_{j} \stackrel{\mathcal{L}}{=} B_{j}\left(G_{j}(1)\right)$. Thus, $Y_{j}$ is a Normal Mean Variance mixture, with mixing distribution the marginal distribution of $\boldsymbol{G}$. Proposition 2.1 states that $\boldsymbol{G}$ is self-decomposable, thus its marginal distributions are self-decomposable. Then, the proof follows easily from Theorem 1.1 in Sato (1999).

We now can define a Sato version of the $\rho \alpha$-models.

Definition 2.2. Let $\boldsymbol{Y}(t)$ be a $\rho \alpha$-model defined in 1.1 with self-decomposable marginal laws. The $\mathbb{R}^{n}$-valued $\rho \alpha$-Sato model $\boldsymbol{Y}^{S}(t)$ is defined by:

$$
\boldsymbol{Y}^{S}(t):=\underline{\mathcal{L}} t^{\boldsymbol{h}} \boldsymbol{Y}(1):=\left(\begin{array}{c}
t^{h_{1}}\left(B_{1}\left(X_{1}(1)\right)+B_{1}^{\rho}(Z(1))\right) \\
\ldots \\
t^{h_{n}}\left(B_{n}\left(X_{n}(1)\right)+B_{n}^{\rho}(Z(1))\right)
\end{array}\right)
$$

where $\boldsymbol{h}=\left(h_{1}, \ldots, h_{n}\right)$ is the self-similar exponent and $t^{\boldsymbol{h}}=\left(t^{h_{1}}, \ldots, t^{h_{n}}\right)$.

By Proposition 2.2 for any choice of $X_{j}$ and $Z$ with self-decomposable laws, the $\rho \alpha$-Sato model has Sato marginal processes. If $\boldsymbol{\rho}=I$, then we have the subclass of $\alpha$ Sato models introduced by Guillaume (2012). Correlations in $\rho \alpha$-Sato models are time independent, and have the same expression as in the $\rho \alpha$-models, reported in Appendix A.

The characteristic function of $\boldsymbol{Y}^{S}(t)$ is given by

$$
\psi_{\mathbf{Y}^{S}(t)}(\boldsymbol{u})=E\left[e^{i \boldsymbol{u} \cdot \boldsymbol{Y}^{S}(t)}\right]=E\left[e^{i\left(\boldsymbol{u} \circ t^{\boldsymbol{h}}\right) \cdot \boldsymbol{Y}(1)}\right]=\psi_{\boldsymbol{Y}(1)}\left(\boldsymbol{u} \circ t^{\boldsymbol{h}}\right)
$$

where $\boldsymbol{u} \circ t^{\boldsymbol{h}}=\left(u_{1} t^{h_{1}}, \ldots, u_{n} t^{h_{n}}\right)$ denotes the Hadamard product and $\psi_{\boldsymbol{Y}}(\boldsymbol{u})$ is provided in A.1. The marginal characteristic functions follow easily:

$$
\psi_{\mathbf{Y}_{j}^{S}(t)}(u)=\psi_{Y_{j}(1)}\left(u t^{h_{j}}\right) .
$$

Using the characteristic function, we can find moments and comoments of a $\rho \alpha$-Sato process, using the relation

$$
E\left[\left(Y_{j}^{S}(t)\right)^{k}\right]=\left.\frac{\psi_{Y_{j}^{S}(t)}^{(k)}(u)}{i^{k}}\right|_{u=0}
$$


where the superscript $(k)$ indicates the $k$-th derivative. By substituting 2.6 in 2.7 we have

$$
E\left[\left(Y_{j}^{S}(t)\right)^{k}\right]=\left.\frac{\psi_{Y_{j}^{S}(t)}^{(k)}(u)}{i^{k}}\right|_{u=0}=\left.\frac{1}{i^{k}} \frac{d^{k}}{d u} \phi_{Y_{j}(1)}\left(u t^{h_{j}}\right)\right|_{u=0}=E\left[\left(Y_{j}(1)\right)^{k}\right] t^{h_{j} k}
$$

Therefore

$$
\begin{aligned}
E\left[Y_{j}^{S}(t)\right] & =E\left[Y_{j}(1)\right] t^{h_{j}}, \\
\operatorname{Var}\left[Y_{j}^{S}(t)\right] & =\operatorname{Var}\left[Y_{j}(1)\right] t^{2 h_{j}}, \\
\operatorname{Skew}\left[Y_{j}^{S}(t)\right] & =\frac{E\left[\left(Y_{j}^{S}(t)-E\left[Y_{j}^{S}(t)\right]\right)^{3}\right]}{\operatorname{Var}\left[Y_{j}^{S}(t)\right]^{3 / 2}}=\operatorname{Skew~}\left[Y_{j}(1)\right], \\
\operatorname{Kurt}\left[Y_{j}^{S}(t)\right] & =\frac{E\left[\left(Y_{j}^{S}(t)-E\left[Y_{j}^{S}(t)\right]\right)^{4}\right]}{\operatorname{Var}\left[Y_{j}^{S}(t)\right]^{2}}=\operatorname{Kurt}\left[Y_{j}(1)\right] .
\end{aligned}
$$

It is clear that marginal skewness and marginal kurtosis of the $\rho \alpha$-Sato process, unlike $\rho \alpha$-Lévy process, are constant over time. This can be a reasonable assumption when modeling asset returns, up to a certain time to maturity, as observed in Madan and Schoutens (2013).

We do not analyse in this paper the joint dynamics of the $\rho \alpha$-Sato processes, but we empirically investigate its global fit on market data. Nevertheless, we conclude this section with a complete picture of the multivariate Sato processes and their relationship with the $\rho \alpha$-Sato models.

The notion of self-decomposability has been extended to the notion of operator selfdecomposability by Urbanik (1978). Preliminarily we have to introduce the following notions. For a set $J \subset \mathbb{R}$, let $M_{J}(n)$ be the set of real $n \times n$ matrices whose eigenvalues have real part in $J \subset \mathbb{R}$. For $a>0$ and an $n \times n$ matrix $Q$ let

$$
a^{Q}=\sum_{n=0}^{\infty} \frac{1}{n !}(\log a)^{n} Q^{n}
$$

We can now give the following definition.

Definition 2.3. Let $Q \in \mathcal{M}_{(0, \infty)}(n)$. A random vector $\boldsymbol{X}$ has $Q$-self-decomposable distribution $\mu$ is, for every $b>1$, there is a $\boldsymbol{X}_{b}$ such that

$$
\boldsymbol{X} \stackrel{\mathcal{L}}{=} b^{-Q} \boldsymbol{X}+\boldsymbol{X}_{b}
$$

If a distribution is $Q$-self-decomposable we can define a multivariate Sato process by scaling the marginal distributions with different scale parameters. In fact Theorem 3.2. in Sato (1991) asserts that given a $Q$-self-decomposable measure $\mu \in \mathbb{R}^{n}$ and a continuous function $b(t)$ on $[0, \infty)$ such that $b(1)=0$ there exists a stochastically continuous process 
$\boldsymbol{X}(t)$ with independent increments such that $\mathcal{L}(\boldsymbol{X}(1))=\mu$. The process is unique in law and $\boldsymbol{X}(t) \stackrel{\mathcal{L}}{=} t^{Q} \boldsymbol{X}(1)+b(t)$. Thus, if $\boldsymbol{X}$ has $Q$-self decomposable distribution, we can introduce a Sato process with different scale parameters as follows:

$$
\boldsymbol{X}(t): \underline{\underline{\mathcal{L}}}\left(\begin{array}{c}
t^{h_{1}} X_{1} \\
\ldots \\
t^{h_{n}} X_{n}
\end{array}\right), h_{j}>0, j=1, \ldots, n .
$$

To introduce $\rho \alpha$-Sato models we have proved that $\rho \alpha$-distributions have self-decomposable marginal distributions. We now briefly address the question of the $\rho \alpha$-distribution self-decomposability at the multivariate level. Obviously, if we consider the independence subcase, obtained if the common component of the subordinator degenerates, $\boldsymbol{Y}$ has $Q$-self-decomposable distribution for $Q=\operatorname{diag}\left(q_{1}, \ldots, q_{n}\right)$ (see Barndorff-Nielsen et al. (2001)). Also in the symmetric case if $\boldsymbol{G}$ is self-decomposable, the $\rho \alpha$-distributions are self-decomposable. In fact, if $\boldsymbol{\mu}=\mathbf{0}$, the multivariate Brownian motions $\boldsymbol{B}(t)$ and $\boldsymbol{B}^{\rho}(t)$ are strictly stable with $\alpha=\frac{1}{2}$. Thus, $\boldsymbol{Y}$ is self-decomposable and also $Q$-selfdecomposable, as shown in Barndorff-Nielsen et al. (2001).

However, in general, the $\rho \alpha$-distribution is not self-decomposable, although the subordinating distribution of $\boldsymbol{G}$ is self-decomposable. In fact, Takano (1989) proved that a multivariate normal distribution subordinated by a generalized gamma distribution is self-decomposable iff the multivariate normal distribution has zero drift. Therefore, if $Z$ belongs to the family of generalized gamma convolutions, the systematic component $\boldsymbol{Y}^{\rho}$ is self-decomposable iff $\boldsymbol{\mu}=\mathbf{0}$. Summing up, if the subordinator components are self-decomposable, the $\rho \alpha$-distributions have self-decomposable marginal distributions and in some limit cases they are also $Q$-self decomposable. Therefore, the $\rho \alpha$-Sato models have marginal processes in the Sato class, but they are Sato processes on $\mathbb{R}^{n}$ in two subcases only: independent components and $\boldsymbol{\mu}=\mathbf{0}$.

\section{Specifications}

We now specify $\rho \alpha$-Sato process $\boldsymbol{Y}$ to have Sato variance gamma (Sato-VG) and Sato normal inverse Gaussian (Sato-NIG) marginal distributions, as in Luciano and Semeraro (2010). The different specifications are obtained from the time one distribution of the $\rho \alpha$-variance gamma ( $\rho \alpha-\mathrm{VG})$ and $\rho \alpha$-normal inverse Gaussian $(\rho \alpha$-NIG) models, which

are recalled in Appendix B. The $\rho \alpha-\mathrm{VG}$ has variance gamma (VG) marginal processes and the $\rho \alpha$-NIG has normal inverse Gaussian (NIG) marginal processes by construction, and these marginal processes are self-decomposable. For each specification we introduce the notation and parameter conditions used in the practical implementation. 


\subsection{Variance Gamma}

Let $\boldsymbol{Y}$ be a $\rho \alpha$-VG as defined in Appendix B.1, then the process $\boldsymbol{Y}^{S}(t)$ in Definition 2.2 (eqn. (2.4)) is a $\rho \alpha$-Sato process with time one marginal distribution of VG type. We call the process $\boldsymbol{Y}^{S} \rho \alpha$-Sato-VG.

The $\rho \alpha$-VG and the $\rho \alpha$-Sato-VG have the same characteristic function at time one, which is

$$
\psi_{\boldsymbol{Y}}(\boldsymbol{u})=\prod_{j=1}^{n}\left(1-\alpha_{j}\left(i \mu_{j} u_{j}-\frac{1}{2} \sigma_{j}^{2} u_{j}^{2}\right)\right)^{-\left(\frac{1}{\alpha_{j}}-a\right)}\left(1-\left(i \boldsymbol{u}^{T} \boldsymbol{\mu}^{\rho}-\frac{1}{2} \boldsymbol{u}^{T} \boldsymbol{\Sigma}^{\rho} \boldsymbol{u}\right)\right)^{-a}
$$

Thus, the $\rho \alpha$-Sato-VG characteristic function at any time $t$ can be derived by applying

(2.5). The $\boldsymbol{Y}^{S} \rho \alpha$-Sato-VG correlations are independent of time, thus they are the same correlations of the $\rho \alpha$-VG process, which are:

$$
\rho_{\boldsymbol{Y}}(i, j)=\frac{\left(\mu_{i} \alpha_{i} \mu_{j} \alpha_{j}+\rho_{i j} \sigma_{i} \sqrt{\alpha_{i}} \sigma_{j} \sqrt{\alpha_{j}}\right)}{\sqrt{\left(\sigma_{i}^{2}+\mu_{i}^{2} \alpha_{i}\right)\left(\sigma_{j}^{2}+\mu_{j}^{2} \alpha_{j}\right)}} a .
$$

They are increasing in $a$, which satisfies the constraint

$$
0<a<\min _{j}\left(\frac{1}{\alpha_{j}}\right)
$$

By setting $\rho_{i j}=0$ for $i \neq j$, we obtain as a subcase the $\alpha$-VG process introduced in Semeraro (2008).

The process $\boldsymbol{Y}$ has a total of $1+3 n+\frac{n(n+1)}{2}$ parameters. It has one common parameter $a$, three marginal parameters $\mu_{i}, \sigma_{i}, \alpha_{i}$ for each marginal distribution, $j=1 ; \ldots, n$; and as many additional parameters as the distinct Brownian motions correlations $\rho_{i j}(i>j), i ; j=1, \ldots, n$.

\subsection{Normal inverse Gaussian}

Let $\boldsymbol{Y}$ be a $\rho \alpha$-NIG process introduced in Appendix B.2, then the process $\boldsymbol{Y}^{S}(t)$ in Definition 2.2 (eqn. (2.4)) is a $\rho \alpha$-Sato process with time one marginal distribution of NIG type. We call the process $\boldsymbol{Y}^{S} \rho \alpha$-Sato-NIG. The $\rho \alpha$-NIG and $\rho \alpha$-Sato-NIG processes have the same characteristic function at time one, which is

$$
\begin{aligned}
\psi_{\boldsymbol{Y}}(\boldsymbol{u}) & =\exp \left\{-\sum_{j=1}^{n}\left(1-\frac{a}{\zeta_{j}}\right)\left(\sqrt{-2\left(i \beta_{j} \delta_{j}^{2} u_{j}-\frac{1}{2} \delta_{j}^{2} u_{j}^{2}\right)+\zeta_{j}^{2}}-\zeta_{j}\right)\right. \\
& \left.-a\left(\sqrt{-2\left(i \boldsymbol{u}^{T} \boldsymbol{\mu}^{\rho}-\frac{1}{2} \boldsymbol{u}^{T} \boldsymbol{\Sigma}^{\rho} \boldsymbol{u}\right)+1}-1\right)\right\},
\end{aligned}
$$

where $\zeta_{j}=\delta_{j} \sqrt{\gamma_{j}^{2}-\beta_{j}^{2}}$. Thus, the $\rho \alpha$-Sato-NIG characteristic function at any time $t$ derives straightforwardly from (3.4) and (2.5). The $\boldsymbol{Y}^{S} \rho \alpha$-Sato-NIG correlations are 
independent of time, thus they are the same correlations of the $\rho \alpha$-NIG process. Setting $\zeta_{j}=\delta_{j} \sqrt{\gamma_{j}^{2}-\beta_{j}^{2}}$, the linear correlations of the $\rho \alpha \mathrm{NIG}$ process are

$$
\rho_{\boldsymbol{Y}}(i, j)=\frac{\beta_{i} \frac{\delta_{i}^{2}}{\zeta_{i}^{2}} \beta_{j} \frac{\delta_{j}^{2}}{\zeta_{j}^{2}}+\rho_{i j} \frac{\delta_{i}}{\zeta_{i}} \frac{\delta_{j}}{\zeta_{j}}}{\sqrt{\left(\gamma_{i}^{2} \delta_{i}\left(\gamma_{i}^{2}-\beta_{i}^{2}\right)^{-\frac{3}{2}}\right)\left(\gamma_{j}^{2} \delta_{j}\left(\gamma_{j}^{2}-\beta_{j}^{2}\right)^{-\frac{3}{2}}\right)}} a
$$

They are increasing in $a$, and, under $(B .1)$ and $(B .2)$, must satisfy the constraint

$$
0<a<\min _{j} \zeta_{j}
$$

By setting $\rho_{i j}=0$ for $i \neq j$, we obtain the $\alpha$-NIG specification.

The process $\boldsymbol{Y}$ has a total of $1+3 n+\frac{n(n-1)}{2}$ parameters, where $a$ is a common parameters; $\beta_{i}, \delta_{i}, \zeta_{i}$ are marginal parameters and $\rho_{i j}(i>j), i, j=1, \ldots, n$ are the correlation cofficients between the Brownian components. As in the VG specification, the number of parameters increses as $n^{2}$, due to the presence of the Brownian motion correlations. Notice that the presence of the $\rho_{i j}$ parameters allows to have negative correlations indipententely from the sign of the drifts. For a theoretical discussion of the role of the Brownian motion correlations in matching linear correlations see Luciano and Semeraro (2010) and Luciano et al. (2016).

Remark 1. We choose to perform the analysis starting from multivariate Lévy processes with popular one dimensional processes, such as VG and NIG processes. Among them we focus on the $\rho \alpha$-models for two main reasons: firstly, they generalize the Sato $\alpha-V G$ process studied in Guillaume (2012), adding correlation flexibility. Secondly, this class of model is easy to calibrate and admits a decoupled calibration. Thus, we can calibrate the marginal parameters for both the Lévy and the corresponding Sato processes and compare the correlation spanned. Other approaches to model dependence - as the one in Marfè (2012) - could be considered, see, e.g., Boen and Guillaume (2016).

Remark 2. We remark that the restrictions enforcing the marginal processes to belong to well-known classes allows $\rho \alpha$-models to be parsimonious in terms of marginal parameters and permits a two-step calibration procedure, providing suitable initial conditions to the joint calibration approach, when required. Without this restriction, the joint procedure becomes mandatory and the computional complexity increases in practice.

\section{Model calibration and correlation flexibility}

In the present section we calibrate the $\rho \alpha$-models to market data, considering four different specifications of the marginal distributions: $\rho \alpha$-VG, $\rho \alpha$-Sato-VG, $\rho \alpha$-Sato-NIG and $\rho \alpha$-Sato-NIG. We first calibrate the marginal distributions of top stocks of the S\&P 
500 to option prices and then explore the linear and nonlinear dependence structure of different model specifications on all possible baskets of given size. The dependence structure of these models allows us to easily find the correlation flexibility of the corresponding $\alpha$-models by simply setting $\rho=0$, we therefore show the improvement of correlation flexibility due to the additional parameter. Finally, we study how nonlinear dependence impact prices of derivative contracts that are well-known to be sensitive to the underlyings' dependence, such as basket, best-of, worst-off and spread options. We do so by performing a regression analysis.

\subsection{Data}

We consider the first 14 constituents of the S\&P 500 Index by market capitalization at October 26, 2016: Apple, Microsoft, Exxon, Johnson\&Johnson, Amazon, Facebook, Berkshire Hathaway (Class B), General Electric, JP Morgan Chase \& Co., Alphabet (Class A), Alphabet (Class C), Procter\&Gamble, AT\&T, Wells Fargo. To make our analysis more robust, we extend the analysis backward in time, considering also quotes on October 31st, 2012 and November 5, 2008. We have 3 different quoting dates, each about every four years, associated to different market volatility levels as measured by the VIX index. According to Figure 1 that shows the performance of the VIX Index from January 2007 to May 2017, we can associate to our quoting dates three different scenarios of market volatility: high volatility (VIX level of $54.56 \%$ as of November 5 , 2008), medium volatility (VIX level of $18.60 \%$ as of October 31st, 2012) and low volatility scenario (VIX level of $14.24 \%$ as of October 26, 2016).

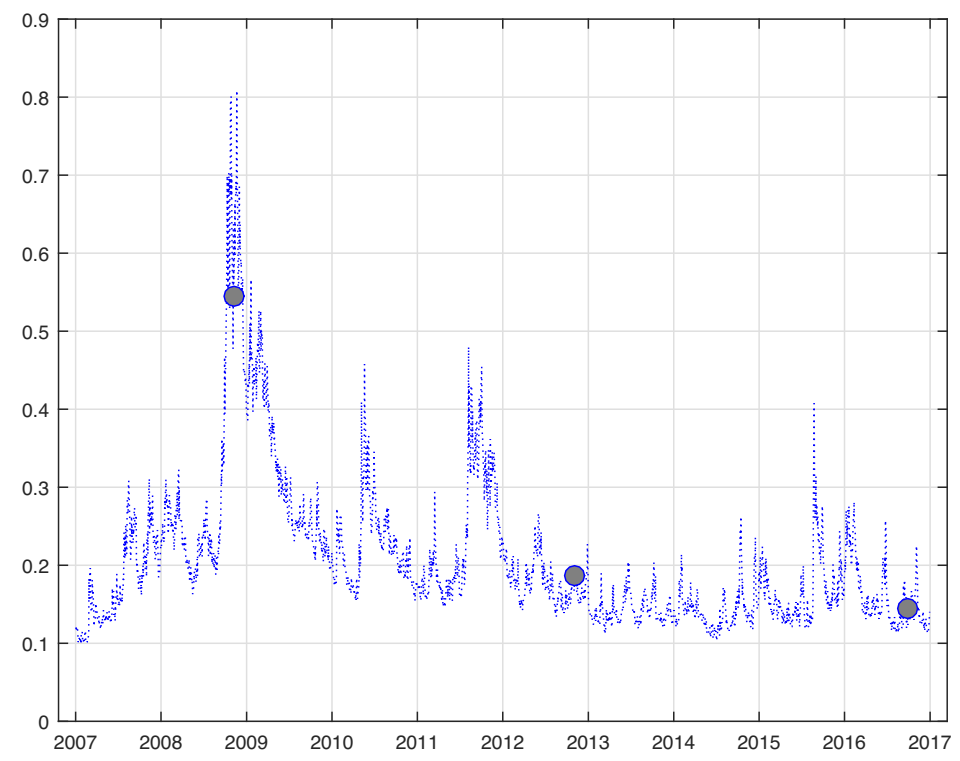

Figure 1: Time series plot of the VIX Index time from January 2007 to December 2016. The grey bullets show our three reference dates: November 5, 2008, October 31st and October 26, 2016. 
Option quotes for all underlyings are collected from Bloomberg, together with interest rates and dividend yields. We recall that Facebook quotes start from May 2012 and Berkshire Hathaway (Class B) quotes start from December 2008. Also, Google Inc. splitted its stocks creating Alphabet (Class A) and Alphabet (Class C) shares in year 2014. Furthermore, dividend yields for Google Inc. were not available for November 5, 2008. Therefore, the dataset for November 5, 2008 consists of 10 stocks: Apple, Microsoft, Exxon, Johnson\&Johnson, Amazon, General Electric, JP Morgan Chase \& Co., Procter\&Gamble, AT\&T, Wells Fargo, while the dataset for October 31st, 2012 consists of 13 stocks: Apple, Microsoft, Exxon, Johnson\&Johnson, Amazon, Facebook, Berkshire Hathaway (Class B), General Electric, JP Morgan Chase \& Co., Google Inc., Procter\&Gamble, AT\&T, Wells Fargo.

\subsection{Marginal fit and dependence structure flexibility}

Let $\boldsymbol{S}=\{\boldsymbol{S}(t), t \geq 0\}$ be a 2 -dimensional price process, $\boldsymbol{S}=\{\boldsymbol{S}(t), t \geq 0\}$, such that

$$
S_{j}(t)=S(0) \frac{e^{\left(r-q_{j}\right) t+Y_{j}(t)}}{\mathbb{E}\left(e^{Y_{j}(t)}\right)}, \quad j=1,2,
$$

where $r$ is the risk-free rate, $q_{j}$ is the dividend yield of asset $j$, and $\boldsymbol{Y}$ is a $\rho \alpha$-process.

Marginal calibrations are performed by minimizing the Root Mean Square Error (RMSE) between model and market implied volatilities

$$
R M S E=\sqrt{\frac{1}{N} \sum_{i=1}^{N}\left(I V_{i}^{m k t}-I V_{i}^{\text {model }}\right)^{2}}
$$

Our datasets consist of out-of-the-money option prices with time to maturity ranging from 20 days to 2.5 years and log-moneyness from -0.4 to 0.4 . Both time to maturity and strike dimensions are considered simultaneously. Market implied volatilities are computed by matching market prices with Black-Scholes American prices computed by the FST pricing formula (Jackson et al. (2007)). We apply the Carr-Madan pricing formula to obtain model prices, and then compute model implied volatilities by inverting the Black-Scholes formula. The optimization is performed using the Differential Evolution algorithm (Storn and Price (1997)). For comparative purposes, we also report the average percentage error ${ }^{1}$ (APE), as additional goodness of fit measure. For completeness, optimal marginal parameters are summarized in Figure 2.

Table 1 shows the average calibration error for our three datasets on different quoting dates. In line with Guillaume (2012) and Eberlein and Madan (2009), Sato models, in their VG and NIG specifications, provide a good comparable fit of plain vanilla markets

\footnotetext{
${ }^{1} A P E=1 / I \bar{V}^{m k t} \sum_{i=1}^{N} \frac{\mid I V_{i}^{m k t}-I V_{i}^{m o d} e l}{N}$, where $I \bar{V}^{m k t}$ is the average market implied volatility on the $N$ options.
} 


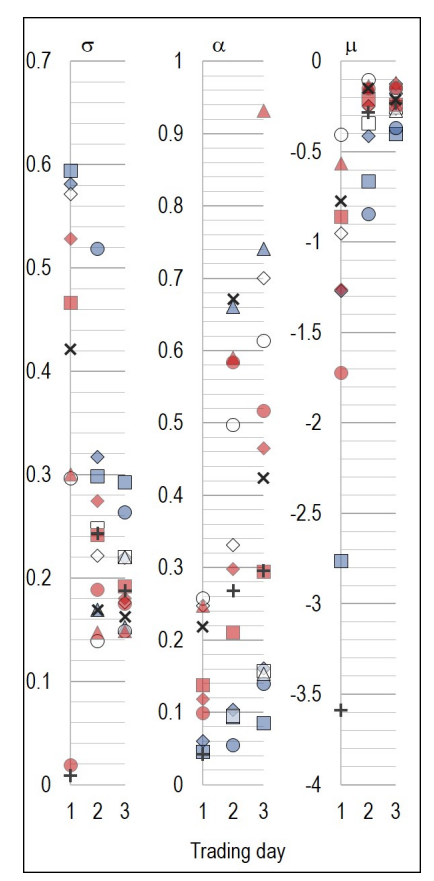

(a) VG

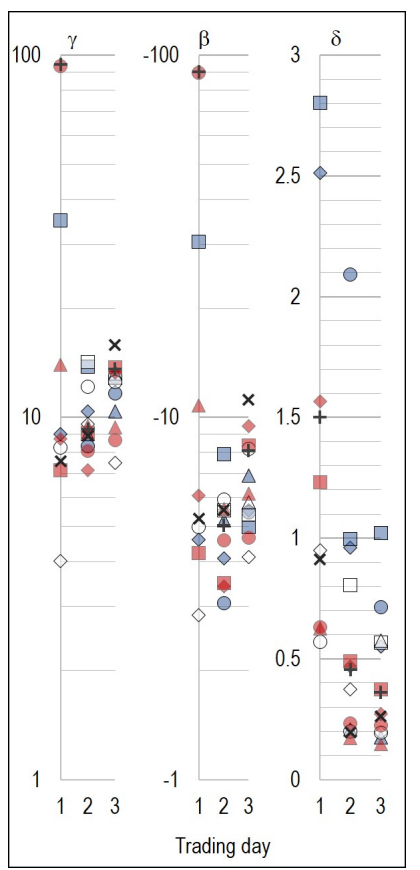

(c) NIG

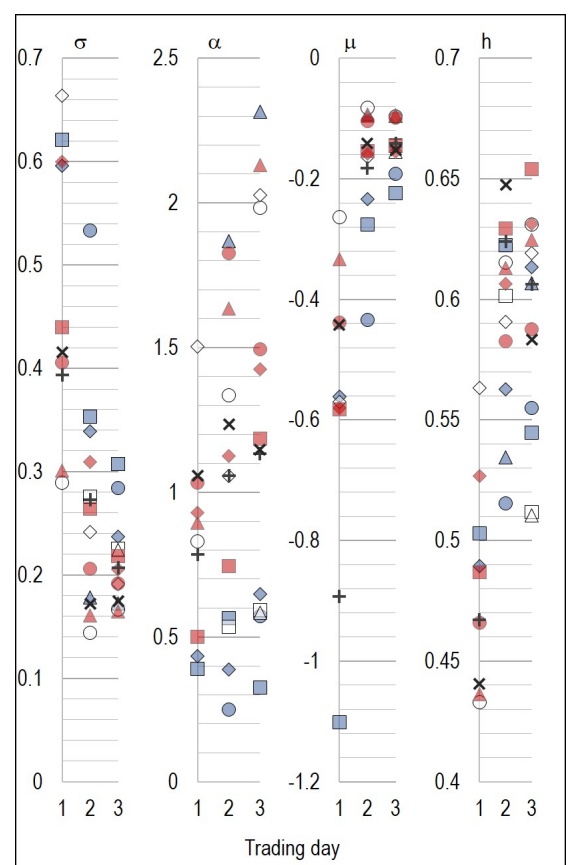

(b) Sato-VG

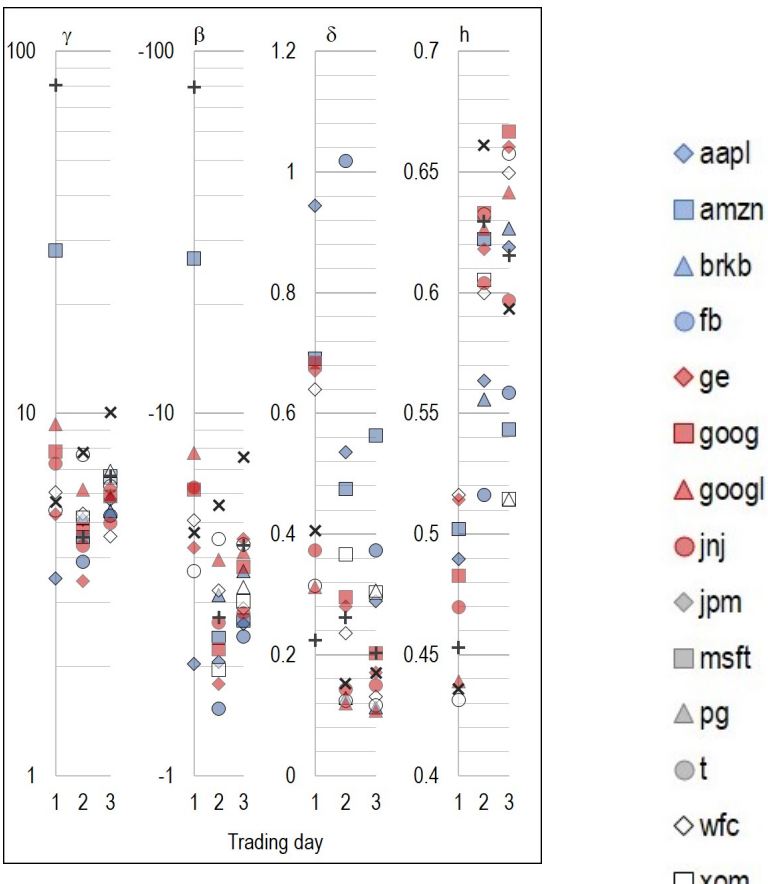

(d) Sato-NIG $\diamond$ aapl

$\mathrm{fb}$

$\Delta$ ge

goog

$\diamond \mathrm{jpm}$

$\checkmark \mathrm{msft}$

$\mathrm{pg}$

$\square$ xom

Figure 2: Marginal parameters of Lévy and Sato models under the two-step calibration procedure. Trading days are November 5, 2008 (date 1), October 31st, 2012 (date 2) and October 26, 2016 (date 3). 
in both strike and time to maturity dimensions, overperforming Lévy models. This is especially true in the high volatility scenario, when Lévy models show very high root mean squared errors and average percentage errors higher than $11 \%$. This evidence confirms that moments dynamics implied by Sato processes are more in line with market data than the ones implied by Lévy processes.

\begin{tabular}{cccc} 
Date & Model & RMSE & APE \\
\hline 1 & VG & 0.067 & 0.111 \\
1 & Sato-VG & 0.016 & 0.024 \\
1 & NIG & 0.067 & 0.111 \\
1 & Sato-NIG & 0.019 & 0.028 \\
\hline 2 & VG & 0.017 & 0.050 \\
2 & Sato-VG & 0.013 & 0.040 \\
2 & NIG & 0.016 & 0.046 \\
2 & Sato-NIG & 0.010 & 0.032 \\
\hline 3 & VG & 0.016 & 0.055 \\
3 & Sato-VG & 0.012 & 0.040 \\
3 & NIG & 0.015 & 0.051 \\
3 & Sato-NIG & 0.009 & 0.031 \\
\hline
\end{tabular}

Table 1: Average calibration error on the marginal fit of first 14 constituents of the S\&P 500 Index by market capitalization on October 26, 2016 (date 3). Figures for November 5, 2008 (date 1) refer to 10 constituents and figures for October 31st, 2012 (date 2) refer to 13 constituents.

Thereafter, we explore the correlation flexibility considering all possible bivariate baskets: 45 baskets for November 5, 2008 (date 1), 78 baskets for October 31st, 2012 (date 2) and 91 baskets for October 26, 2016 (date 3). For each basket we compute the maximum correlation level achievable within each model by setting the common parameters to their maximum level, i.e., $a$ equal to $a_{\max }$ and $\rho_{i j}$ equal to 1 in $(3.2)$ and (3.5). We have $a_{\max }=\min \left(\frac{1}{\alpha_{1}}, \frac{1}{\alpha_{2}}\right)$ in the VG specifications and $a_{\max }=\min \left(\zeta_{1}, \zeta_{2}\right)$ in the NIG specifications, according to (3.3) and (3.6) respectively. Table 2 presents the proportions of all possible bivariate baskets of a given size for which the maximum achievable correlation is lower under the Lévy model than under the corresponding Sato model. All proportions are well above 0.5, ranging between 0.74 and 0.81 . Not only at the marginal level Sato specifications outperform the Lévy ones, but Sato models show higher flexibility in the correlation fit. If we set correlation to levels $0.25,0.5,0.75$ and 0.9 , and compute the percentage of baskets reaching that correlation level, we always get higher percentages in the case of Sato models, as reported in Table 3. This is confirmed by the median of the maximum correlation level achievable for all baskets, shown in the last column of Table 3. Again, in a high volatility period such as date 1, Sato models are remarkably more flexible than Lévy models, being the median $0.77(0.74)$ in the Sato-VG (Sato-NIG) model vs 0.56 (0.53) of the VG (NIG) model.

Results in Table 1 and 2 taken together suggest that the extra parameter of $\rho \alpha$-Sato 


\begin{tabular}{ccc} 
Date & Model & Proportion \\
\hline 1 & VG & $75.56 \%$ \\
1 & NIG & $77.78 \%$ \\
\hline 2 & VG & $78.21 \%$ \\
2 & NIG & $80.77 \%$ \\
\hline 3 & VG & $74.73 \%$ \\
3 & NIG & $75.82 \%$ \\
\hline
\end{tabular}

Table 2: Proportions of all possible bivariate baskets of a given size for which the maximum achievable correlation is lower under the Lévy model than under the corresponding Sato model. Quoting dates are November 5, 2008 (date 1), October 31st, 2012 (date 2) and October 26, 2016 (date 3).

\begin{tabular}{ccccccc} 
Date & Model & 0.25 & 0.5 & 0.75 & 0.9 & Median \\
\hline 1 & VG & $97.78 \%$ & $55.56 \%$ & $20.00 \%$ & $15.56 \%$ & 0.56 \\
1 & Sato-VG & $100.00 \%$ & $100.00 \%$ & $51.11 \%$ & $22.22 \%$ & 0.77 \\
1 & NIG & $93.33 \%$ & $60.00 \%$ & $17.78 \%$ & $4.44 \%$ & 0.53 \\
1 & Sato-NIG & $100.00 \%$ & $93.33 \%$ & $48.89 \%$ & $13.33 \%$ & 0.74 \\
\hline 2 & VG & $100.00 \%$ & $70.51 \%$ & $29.49 \%$ & $16.67 \%$ & 0.64 \\
2 & Sato-VG & $100.00 \%$ & $85.90 \%$ & $47.44 \%$ & $16.67 \%$ & 0.74 \\
2 & NIG & $100.00 \%$ & $70.51 \%$ & $32.05 \%$ & $15.38 \%$ & 0.61 \\
2 & Sato-NIG & $100.00 \%$ & $87.18 \%$ & $44.87 \%$ & $16.67 \%$ & 0.72 \\
\hline 3 & VG & $100.00 \%$ & $74.73 \%$ & $35.16 \%$ & $14.29 \%$ & 0.68 \\
3 & Sato-VG & $100.00 \%$ & $89.01 \%$ & $38.46 \%$ & $18.68 \%$ & 0.71 \\
3 & NIG & $100.00 \%$ & $74.73 \%$ & $34.07 \%$ & $13.19 \%$ & 0.64 \\
3 & Sato-NIG & $100.00 \%$ & $84.62 \%$ & $38.46 \%$ & $15.38 \%$ & 0.71 \\
\hline
\end{tabular}

Table 3: Percentage of bivariate baskets whose maximum achievable correlation is higher or equal to levels $0.25,0.5,0.75$ and 0.9 , for all quoting dates and models. The last column shows the median of the maximum achievable correlations.

models allows a more accurate marginal fit, while enhancing on average the correlation flexibility with respect to Lévy specifications. This is examined in more detail in the next section, where we consider also how increasing the basket size affects the correlation fit.

\subsection{Two-step calibration procedure}

By increasing the number of assets the constraint on the common parameter $a$ becomes more binding, as one can see from equation (3.3) for the VG case and from Equation (3.6) for the NIG case. These bounds reduce the correlation ranges of each pair of assets. A measure of this effect is a challenging issue, since we should define a range for a correlation matrix. Furthermore, the Brownian correlation parameters should be also considered to define this range. We therefore adopt a different approach, by using the historical correlation matrix as a proxy of asset correlations for illustration purposes. 
Ideally, factor-based Lévy processes should be calibrated to the market prices of multi-asset derivatives. However, this approach is not always feasible in practice. Therefore, we explore the model flexibility by calibrating the correlation structure to historical correlations, as in Luciano and Schoutens (2006) and Leoni and Schoutens (2008). An alternative, more robust approach, can be found in Guillaume (2012). Summary statistics of the historical correlation matrices are reported in Table 4 . For each trading day, the table provides the minimum, the maximum, the mean and the median of all pairwise correlation values. Historical correlations have been computed on daily log returns by exponentially-weighted moving average over the previous year.

\begin{tabular}{ccccc} 
Date & Min & Max & Mean & Median \\
\hline 1 & 0.46 & 0.89 & 0.67 & 0.68 \\
2 & -0.24 & 0.77 & 0.30 & 0.33 \\
3 & -0.39 & 0.98 & 0.28 & 0.30 \\
\hline
\end{tabular}

Table 4: Summary statistics of the historical correlation matrix computed on daily log returns by exponentially-weighted moving average over the previous year. Quoting dates are November 5, 2008 (date 1), October 31st, 2012 (date 2) and October 26, 2016 (date $3)$.

We calibrate the dependence structure for each basket of underlyings by minimising the root mean squared error between empirical and model return correlations, considering baskets with sizes ranging from two to five.

The number of all possible baskets of different sizes analyzed in our two step calibration procedure is reported in Table 5 .

\begin{tabular}{cccc} 
Size & Date 1 & Date 2 & Date 3 \\
\hline 2 & 45 & 78 & 91 \\
3 & 120 & 286 & 364 \\
4 & 210 & 715 & 1001 \\
5 & 252 & 1287 & 2002 \\
\hline
\end{tabular}

Table 5: Number of all possible baskets of any given size (from 2 to 5 ) on November 5 , 2008 (date 1), October 31st, 2012 (date 2) and October 26, 2016 (date 3).

For different models, volatility scenarios and basket sizes, Figures 3,4 and 5 show the proportion of baskets whose correlation maximum absolute error (MAE) is higher than any given level. More specifically, the correlation MAE is defined as

$$
\max _{1 \leq i, j \leq n}\left|\rho_{Y}^{e m p}(i, j)-\rho_{Y}(i, j)\right|,
$$

where $\rho_{\boldsymbol{Y}}^{e m p}(i, j)$ and $\rho_{\boldsymbol{Y}}(i, j)$ are the sample and model return correlations, respectively. 

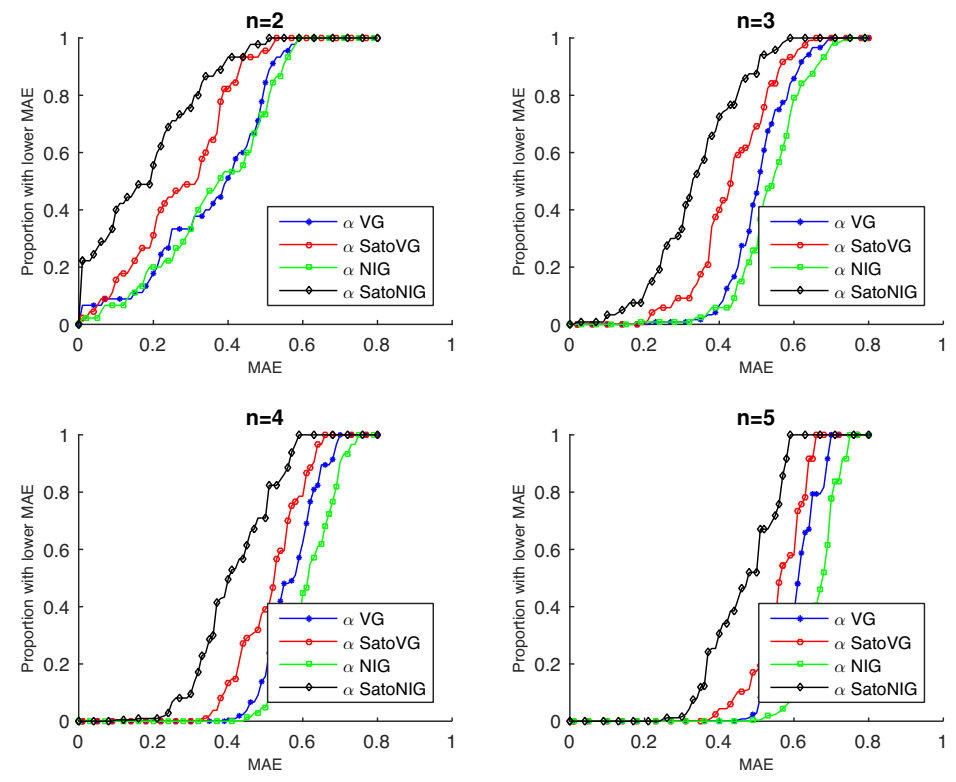

(a) $\alpha$ models
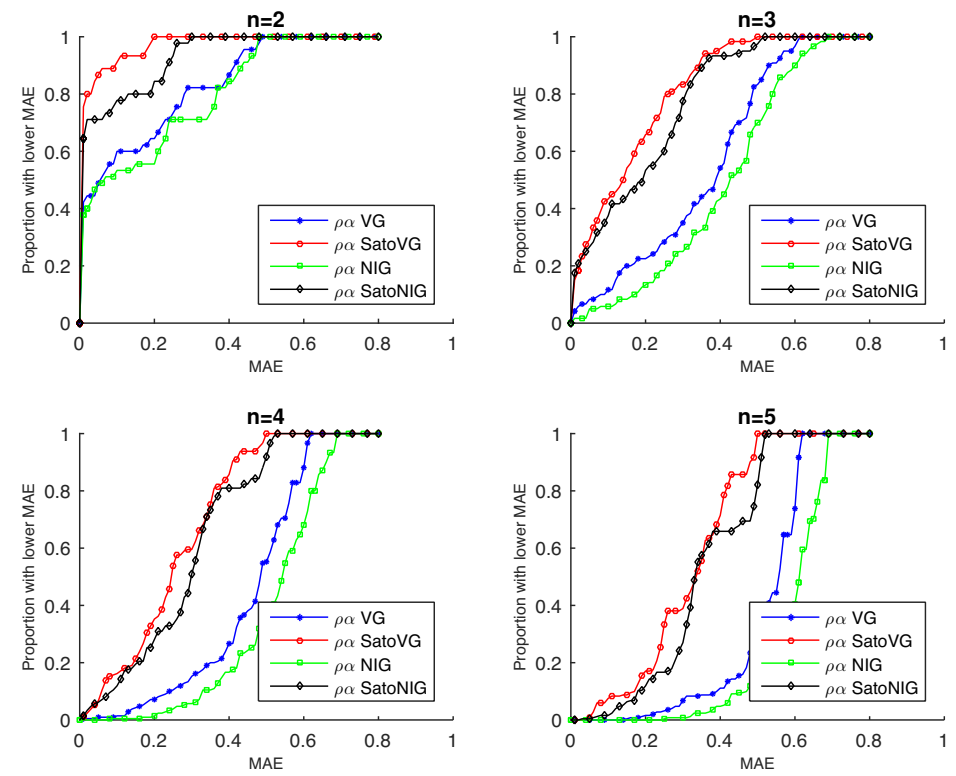

(b) $\rho \alpha$ models

Figure 3: Proportion of baskets whose correlation maximum absolute error (MAE) is higher than any given level, for different models and basket sizes $n=2,3,4,5$ on November 5, 2008. 

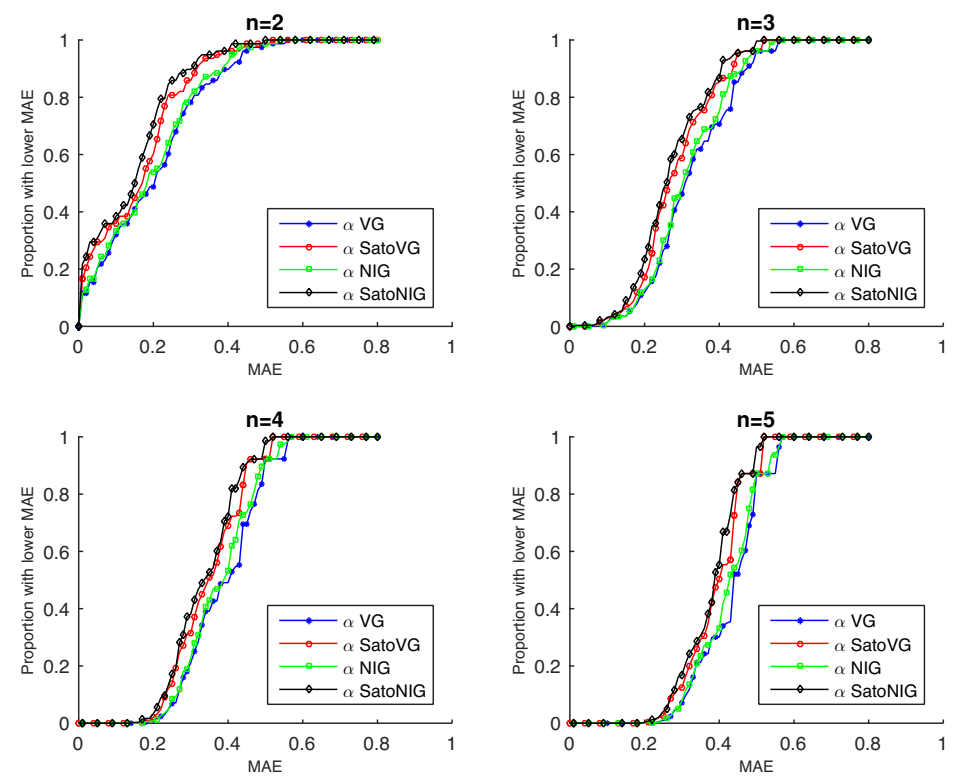

(a) $\alpha$ models
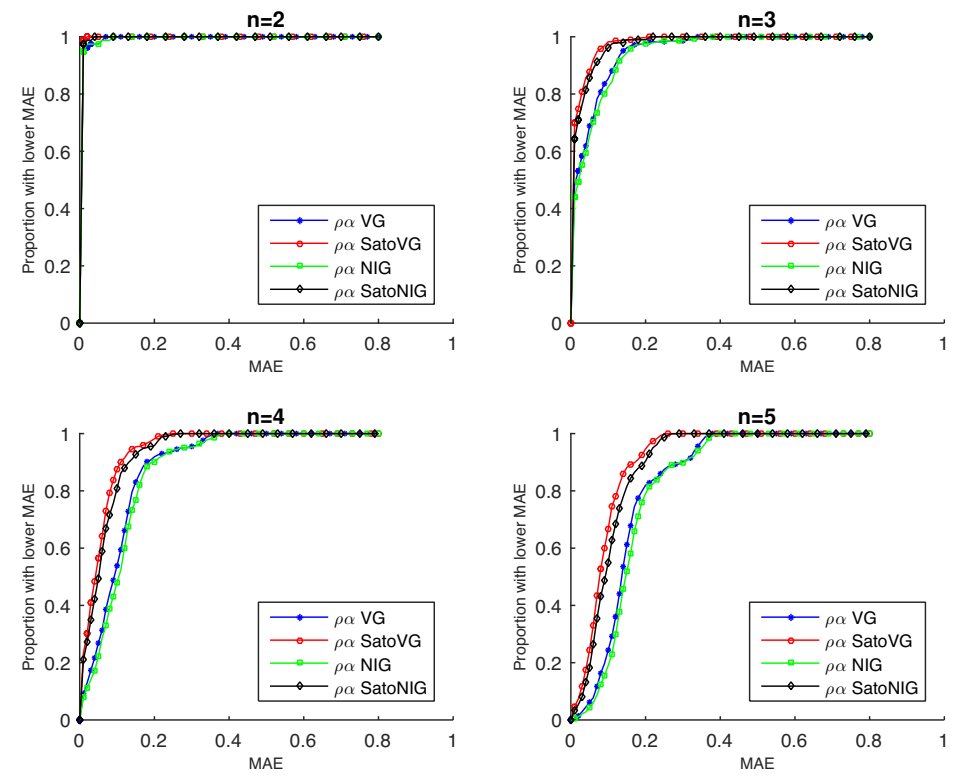

(b) $\rho \alpha$ models

Figure 4: Proportion of baskets whose correlation maximum absolute error (MAE) is higher than any given level, for different models and basket sizes $n=2,3,4,5$ on October 31, 2012. 

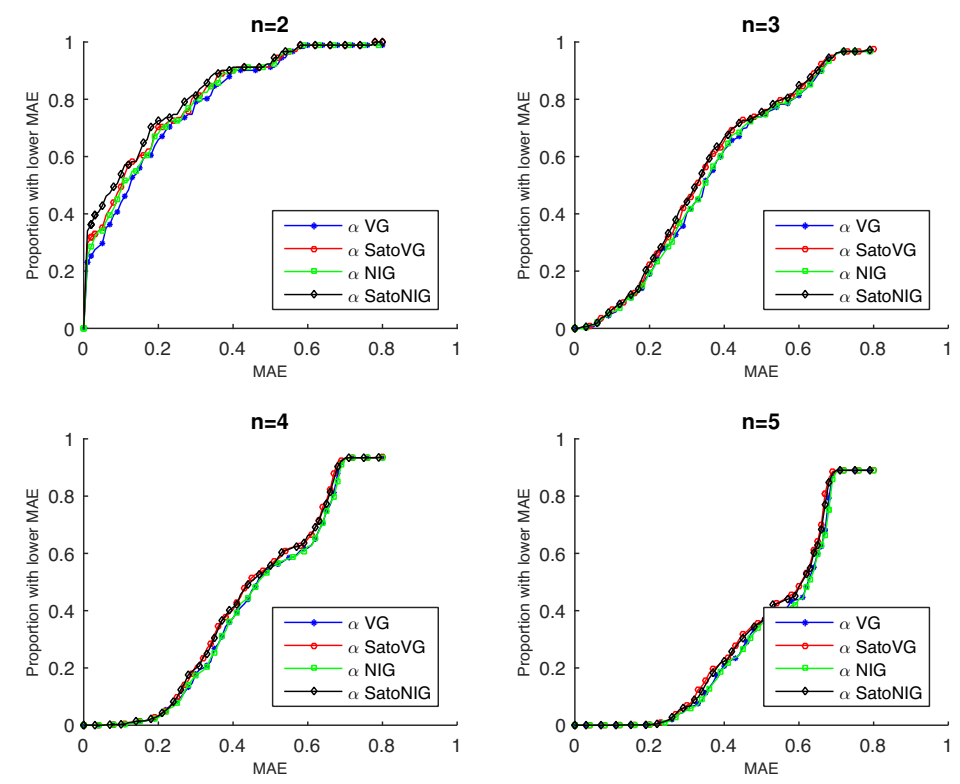

(a) $\alpha$ models
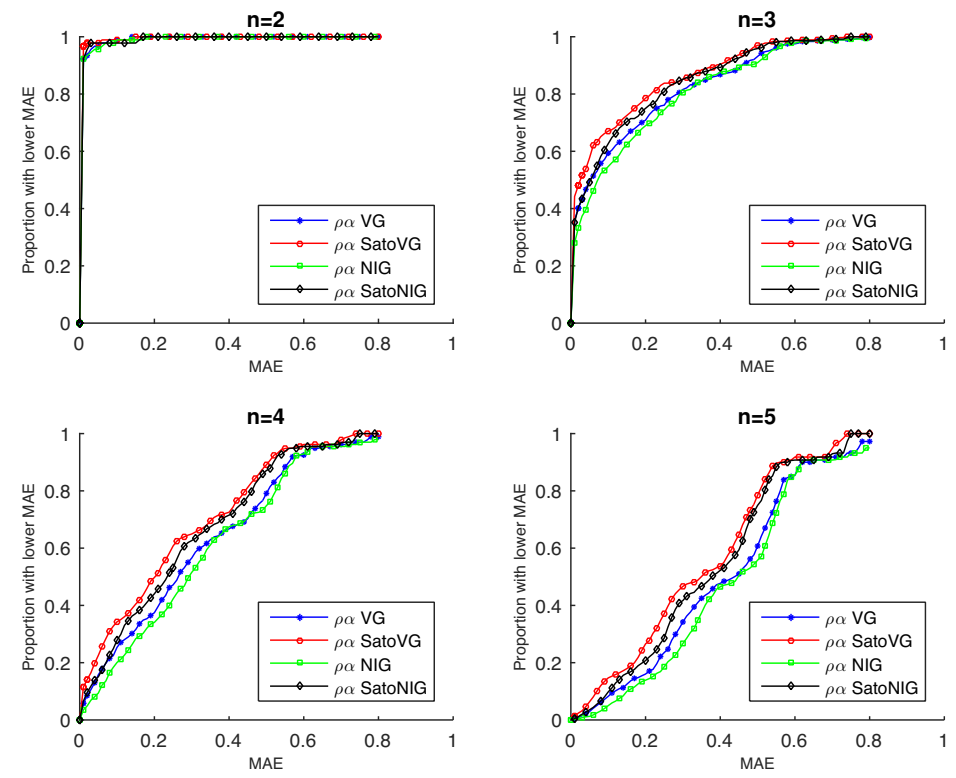

(b) $\rho \alpha$ models

Figure 5: Proportion of baskets whose correlation maximum absolute error (MAE) is higher than any given level, for different models and basket sizes $n=2,3,4,5$ on October $26,2016$. 
We can see that in all cases the Sato models outperform the Lévy counterpart. This effect is more pronounced in a high volatility market, as of November 5, 2008. It is also evident that the $\rho \alpha$-models significantly improve the correlation fit of the $\alpha$-models, thanks to the additional Brownian correlation parameters.

We underline that the same correlations can be fitted by different pairs of common parameters $\left(a, \Sigma^{\rho}\right)$. This fact will be used in the next section to show that in principle the model is able to have different nonlinear dependence structures for a given correlation level when correlation levels are not too binding. A nonlinear dependence measure could be used to identify $a$ and the Brownian motion correlations in general.

Finally, Table 6 shows correlation, cokurtosis and coskewness ranges spanned by Sato and Lévy models with the parameters calibrated on October 26, 2016 (date 3) for all possible baskets generated by the first five assets. Specifically, we consider the standardized coefficients

$$
\begin{gathered}
m_{r s}=\frac{\mathbb{E}\left[\left(R_{1}(t)-\mathbb{E}\left(R_{1}(t)\right)\right)^{r}\left(R_{2}(t)-\mathbb{E}\left(R_{2}(t)\right)\right)^{s}\right]}{\operatorname{Var}\left[R_{1}(t)\right]^{r / 2} \operatorname{Var}\left[R_{2}(t)\right]^{s / 2}}, \\
(r, s) \in(1,2),(2,1),(1,3),(3,1),(2,2),
\end{gathered}
$$

with $R_{i}(t)=\log \frac{S_{i}(t)}{S_{i}(0)}, i=1,2$, for $t$ equal to 1 month, 6 months and 1 year. While correlation is time independent in both models, comoments are time independent in the $\rho \alpha$-Sato models and time-dependent in the $\rho \alpha$-Lévy models. For sake of brevity, only the cokurtosis $m_{22}$ and the coskewness $m_{12}$ are reported in Table $\mathbf{6}$, which also shows the maximum value of $a$. Notice that the constraint on the common parameter $a$ is more binding in the Sato models, although we observe more often a wider correlation range. This fact indicates that the improvement of the performance of the Sato models is not directly linked to the common parameter $a$, but it derives from the combined influence of marginal parameters on the correlation structure. Cokurtosis ranges clearly indicates the presence of nonlinear dependence, that in Sato models is constant over time, while in Lévy models is higher in the short term and tends to Gaussian values as time to maturity increases. We also observe that the cokurtosis level of Sato models is close to the 3-month cokurtosis level of the Lévy counterpart.

As already highlighted in Guillaume (2012) for the $\alpha$-VG model and confirmed in Marena et al. (2015) for the $\rho \alpha$-VG and $\rho \alpha$-NIG models, a joint calibration approach enable to exploit the trade-off between marginal distributions and correlation fit may be required to enhance the goodness-of-fit of the correlation structure, especially when the number of the assets is large. We complete our empirical analysis by performing a joint calibration procedure. 


\begin{tabular}{|c|c|c|c|c|c|c|c|c|c|}
\hline \multirow{3}{*}{$\begin{array}{l}\text { NIG } \\
\text { basket }\end{array}$} & \multicolumn{9}{|c|}{ Lévy models } \\
\hline & \multirow[t]{2}{*}{$a_{\max }$} & \multicolumn{2}{|c|}{$\rho_{\text {asset }}$} & \multicolumn{3}{|c|}{$\left.\max \left(\left|m_{12}\right|\right)\right)$} & \multicolumn{3}{|c|}{$\max \left(m_{22}\right)$} \\
\hline & & $\min$ & $\max$ & $1 \mathrm{M}$ & $3 \mathrm{M}$ & $6 \mathrm{M}$ & $1 \mathrm{M}$ & $3 \mathrm{M}$ & $6 \mathrm{M}$ \\
\hline aapl/amzn & 6.32 & -0.48 & 0.72 & 0.86 & 0.49 & 0.35 & 6.94 & 3.66 & 2.44 \\
\hline aapl/jnj & 1.84 & -0.21 & 0.52 & 2.34 & 1.35 & 0.96 & 13.51 & 5.53 & 2.54 \\
\hline $\mathrm{aapl} / \mathrm{msft}$ & 4.07 & -0.36 & 0.79 & 2.24 & 1.29 & 0.91 & 13.83 & 6.10 & 3.20 \\
\hline aapl/xom & 2.94 & -0.23 & 0.64 & 2.44 & 1.41 & 1.00 & 14.15 & 5.94 & 2.86 \\
\hline jnj/amzn & 1.84 & -0.17 & 0.37 & 0.53 & 0.31 & 0.22 & 7.02 & 3.19 & 1.75 \\
\hline msft/amzn & 4.07 & -0.29 & 0.56 & 0.77 & 0.45 & 0.31 & 7.22 & 3.49 & 2.09 \\
\hline msft/jnj & 1.84 & -0.14 & 0.67 & 3.28 & 1.90 & 1.34 & 24.75 & 9.52 & 3.81 \\
\hline msft/xom & 2.94 & -0.11 & 0.84 & 3.45 & 1.99 & 1.41 & 26.29 & 10.38 & 4.41 \\
\hline xom/amzn & 2.94 & -0.19 & 0.45 & 0.69 & 0.40 & 0.28 & 7.31 & 3.38 & 1.90 \\
\hline xom/jnj & 1.84 & -0.06 & 0.79 & 4.06 & 2.34 & 1.66 & 37.01 & 13.83 & 5.14 \\
\hline \multicolumn{10}{|l|}{$\mathrm{VG}$} \\
\hline aapl/amzn & 6.23 & -0.49 & 0.73 & 0.82 & 0.47 & 0.33 & 6.01 & 3.37 & 2.39 \\
\hline aapl/jnj & 1.63 & -0.23 & 0.50 & 2.03 & 1.17 & 0.83 & 9.77 & 4.25 & 2.19 \\
\hline $\mathrm{aapl} / \mathrm{msft}$ & 3.40 & -0.37 & 0.73 & 1.95 & 1.13 & 0.80 & 10.22 & 4.78 & 2.75 \\
\hline aapl/xom & 2.36 & -0.25 & 0.59 & 2.09 & 1.21 & 0.85 & 10.03 & 4.48 & 2.40 \\
\hline jnj/amzn & 1.63 & -0.19 & 0.36 & 0.47 & 0.27 & 0.19 & 5.42 & 2.65 & 1.60 \\
\hline msft/amzn & 3.40 & -0.30 & 0.53 & 0.66 & 0.38 & 0.27 & 5.68 & 2.93 & 1.90 \\
\hline msft/jnj & 1.63 & -0.22 & 0.69 & 2.95 & 1.71 & 1.21 & 18.54 & 7.48 & 3.34 \\
\hline msft/xom & 2.36 & -0.22 & 0.83 & 3.04 & 1.75 & 1.24 & 19.19 & 7.98 & 3.77 \\
\hline xom/amzn & 2.36 & -0.21 & 0.42 & 0.57 & 0.33 & 0.23 & 5.54 & 2.75 & 1.71 \\
\hline xom/jnj & 1.63 & -0.17 & 0.83 & 3.67 & 2.12 & 1.50 & 27.35 & 10.70 & 4.46 \\
\hline
\end{tabular}

\begin{tabular}{|c|c|c|c|c|}
\hline \multicolumn{5}{|c|}{ Sato models } \\
\hline$a_{\max }$ & \multicolumn{2}{|c|}{$\rho_{\text {asset }}$} & $\max \left(\left|m_{12}\right|\right)$ & $\max \left(m_{22}\right)$ \\
\hline & $\min$ & $\max$ & & \\
\hline 1.49 & -0.42 & 0.66 & 0.45 & 3.39 \\
\hline 0.53 & -0.20 & 0.57 & 1.46 & 6.09 \\
\hline 0.92 & -0.32 & 0.77 & 1.39 & 6.46 \\
\hline 1.13 & -0.22 & 0.81 & 1.52 & 6.87 \\
\hline 0.53 & -0.15 & 0.37 & 0.31 & 3.07 \\
\hline 0.92 & -0.23 & 0.50 & 0.40 & 3.24 \\
\hline 0.53 & -0.09 & 0.75 & 2.09 & 11.07 \\
\hline 0.92 & -0.03 & 0.89 & 1.80 & 10.26 \\
\hline 1.13 & -0.17 & 0.52 & 0.46 & 3.38 \\
\hline 0.53 & 0.03 & 0.68 & 2.00 & 10.09 \\
\hline 1.54 & -0.47 & 0.71 & 0.46 & 3.28 \\
\hline 0.50 & -0.24 & 0.56 & 1.21 & 4.44 \\
\hline 0.84 & -0.35 & 0.73 & 1.16 & 4.86 \\
\hline 0.87 & -0.27 & 0.72 & 1.24 & 4.88 \\
\hline 0.50 & -0.19 & 0.39 & 0.30 & 2.65 \\
\hline 0.84 & -0.28 & 0.51 & 0.37 & 2.86 \\
\hline 0.50 & -0.20 & 0.77 & 1.76 & 7.88 \\
\hline 0.84 & -0.19 & 0.98 & 1.75 & 8.49 \\
\hline 0.87 & -0.22 & 0.50 & 0.40 & 2.85 \\
\hline 0.50 & -0.10 & 0.76 & 1.79 & 7.91 \\
\hline
\end{tabular}

Table 6: Ranges of correlation, coskewness $m_{12}$ and cokurtosis $m_{22}$ for all bivariate baskets generated by the first five assets. Coskewness and cokurtosis of Lévy models refer to 1-month, 3-months and 1 year time to maturity. Parameters are calibrated on October 26, 2016 (date 3).

\subsection{Joint calibration procedure}

Setting the tolerance on the maximum absolute error in matching asset correlations to $\epsilon$, we fit all option surfaces together. In particular, for each basket of $n$ underlyings, we numerically solve the problem:

$$
\begin{array}{ll}
\min _{\{\boldsymbol{\theta}, a, \boldsymbol{\rho}\}} & \sum_{i=1}^{n} R M S E_{i} \\
\text { s.t. } & \max \left|\rho_{\boldsymbol{Y}}^{e m p}(j, k)-\rho_{\boldsymbol{Y}}(j, k)\right| \leq \epsilon, j \neq k,
\end{array}
$$

where $\boldsymbol{\theta}$ is the vector of all marginal parameters and $\boldsymbol{\rho}=\left\{\rho_{i j}, i=1, \ldots n, j=2, \ldots n\right\}$ are the correlation coefficients between the Brownian components collected in $\boldsymbol{B}^{\rho}$. The relative importance of marginal versus correlation fit can then be fine-tuned through the threshold $\epsilon$. Initial conditions are provided by the parameters of the two step calibration. In our exercise, for all dates and models, we set $\epsilon=0.1$ and we consider all possible baskets generated by the first five assets: 10 baskets of size 2 and 3, 5 baskets of size 4 and one basket of size 5 . Only baskets for which the two-step calibration procedure provides a maximum correlation error higher than 0.1 are considered. Results are summarized in Figure 8 that shows the average RMSE of the two-step calibration vs the 
joint one for each basket. Most baskets are quite closed to the diagonal, implying that the increase in the RMSE of the joint calibration with respect to the two-step procedure is within reasonable values. Furthermore, all large errors of both calibration procedures are associated to Lévy models.

\section{Impact of the nonlinear dependence structure on option prices}

In this section we explore the impact of nonlinear dependence and its evolution over time on two-assets derivatives pricing, under the $\rho \alpha$-Sato and $\rho \alpha$-models. We consider the following derivative contracts: basket, best-off, worst-off and spread options. These contracts are known to be highly sensitive to linear and nonlinear dependence, as highlighted in Tankov (2006) and Marfè (2012). In the bivariate case, we have two common parameters driving the dependence structure within our framework. Therefore, in principle, once calibrated the marginals, we can change nonlinear dependence, leaving the correlation level unchanged. For illustration purposes, Figure 6 shows iso-correlation and iso-comoments contours for the $\rho \alpha$-Sato-NIG process. In fact, the upper right plot in Figure 6 shows the iso-correlation contours for different values of the common parameters: $a$ and the Brownian motion correlation $\rho$. The other subplots, reporting also iso-coskewness and iso-cokurtosis contours, suggest how higher order comoments increase/decrease along the iso-correlation contours. In this sense, the most evident difference between linear and nonlinear dependence can be found when moving along the 0-correlation line. When the 0-line touches the left part of the graphs, the two assets are independent, whilst approaching the right part, the two asset become dependent but still uncorrelated. Then, to quantify the potential impact of nonlinear dependence, we can consider two different model calibrations, representing the minimum and maximum nonlinear dependence scenarios associated to any given asset correlation level. The calibration corresponding to the minimum nonlinear dependence can be obtained by setting the parameter $\rho$ equal to 1 and choosing $a$ according to the iso-correlation contour. On the other hand, if we set the parameter $a=a_{\max }$ and choose $\rho$ according to the iso-correlation contour, we get the maximum nonlinear dependence, as measured by coskewness and cokurtosis.

Figure 7 shows the scatter plots of bivariate returns corresponding to the minimum (upper plot) and maximum (lower plot) nonlinear dependence scenarios, labeled as $A$ and $B$ respectively. These scenarios are associated to a correlation level of 0.5 . The plot also exhibits the iso-price contours for our different contracts, coming into play in our regression exercise. In particular, nonlinear dependence - ceteris paribus - changes the shape of the "point cloud". If an increasing number of points falls on different iso-price curves in the two scenarios, we observe increasing differences in derivative valuations.

We generate by Montecarlo simulation a dataset of options prices written on all 

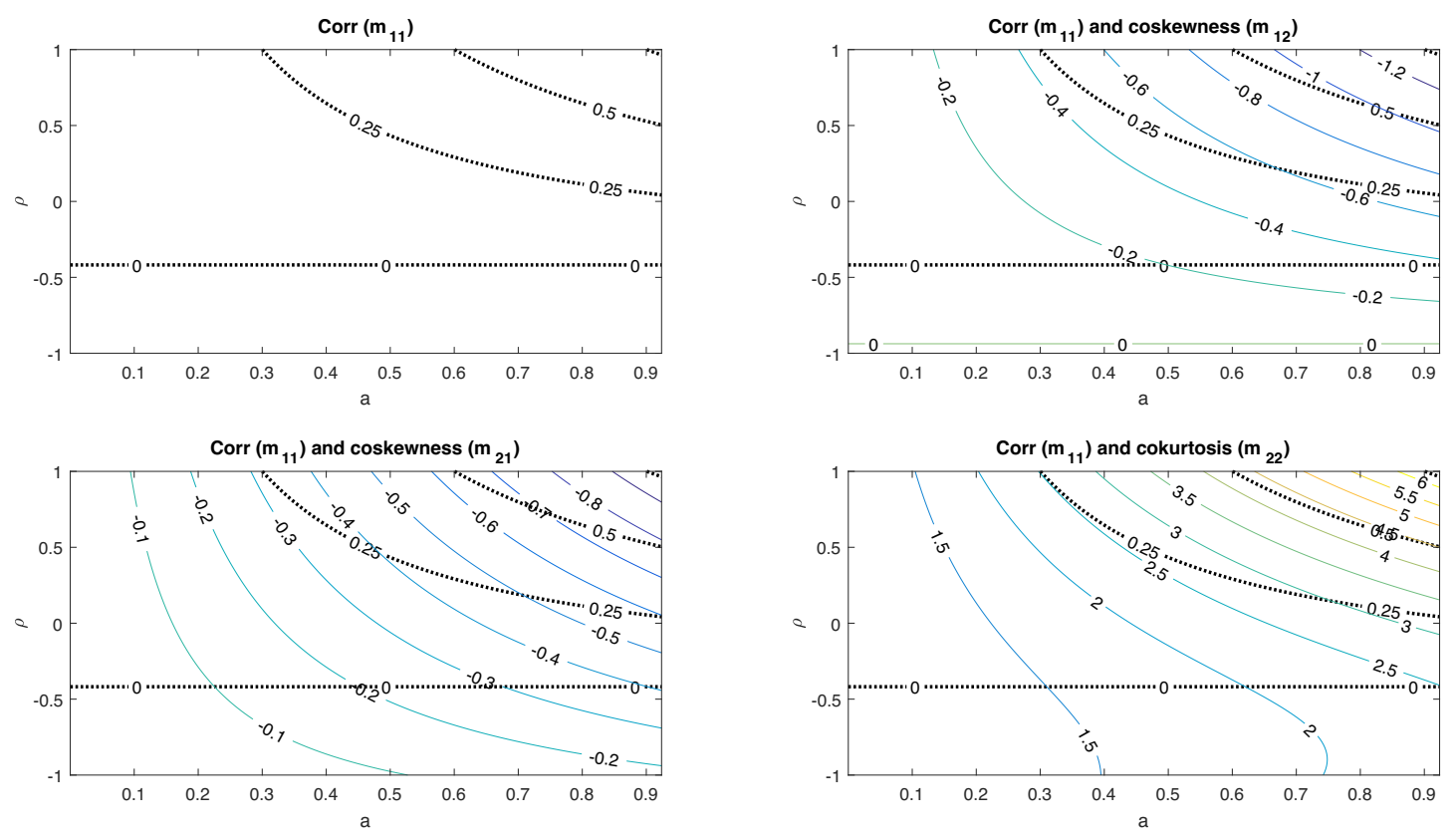

Figure 6: Contour plots for the bivariate basket including Apple and Microsoft on October 26, 2016. Sato-NIG model. Black dot lines indicate iso-correlation contours.

possible bivariate baskets with marginal parameters given by the two step calibration procedure and dependence structures calibrated according to the maximum and minimum dependence scenarios. We thus obtain two set of prices, $P^{A}$ and $P^{B}$. To study the impact of nonlinear dependence on derivative prices, we consider the following regression

$$
\Delta P / P=\beta_{0}+\beta_{1} \operatorname{corr}+\beta_{2} T+\beta_{3} \Delta m_{12}+\beta_{4} \Delta m_{21}+\beta_{5} \Delta m_{13}+\beta_{6} \Delta m_{31}+\beta_{7} \Delta m_{22}+\varepsilon
$$

where $\beta_{0}, \beta_{1}, \ldots, \beta_{7}$ are regression coefficients and $\varepsilon$ is an error term. We define relative price variations as $\Delta P / P=\frac{P^{B}-P^{A}}{P^{A}}$, where $P^{B}$ and $P^{A}$ correspond to values computed under our two different nonlinear dependence scenarios, $A$ and $B$. Within this framework, we have the same correlation in the two different scenarios, i.e. $\operatorname{corr}^{A}=\operatorname{corr}^{B}$, and different absolute values of comoments, i.e. $\left|m_{i j}^{B}\right|>\left|m_{i j}^{A}\right|, i \neq j$. In this sense, the regression analysis can be meaningful in assessing the effect of comoments variations on our relative price variations, keeping all the other relevant factors affecting the price unchanged. We add two control variables: correlation and time to maturity levels, in order to control for possible effects. Therefore, we first run a regression of relative variations in derivative prices $\Delta P / P$ against correlation and time to maturity only. Then, in our second regression, we add comoments variations $\Delta m_{12}, \Delta m_{21}, \Delta m_{13}, \Delta m_{31}$ and $\Delta m_{22}$. In particular, we consider the following correlation levels: $-0.5,-0.25,0,0.25,0.5,0.75$ and 0.9 , and maturities: $1,3,6,12,18,24,30$ months. Table 7 shows the overall number $N$ of baskets considered in the regression experiment for our three different quoting dates and it exhibits the regression results relative to date 3 and the adj. $R^{2}$ relative to date 1 and date 2 . In fact, the significance level of the coefficients is similar for all the 

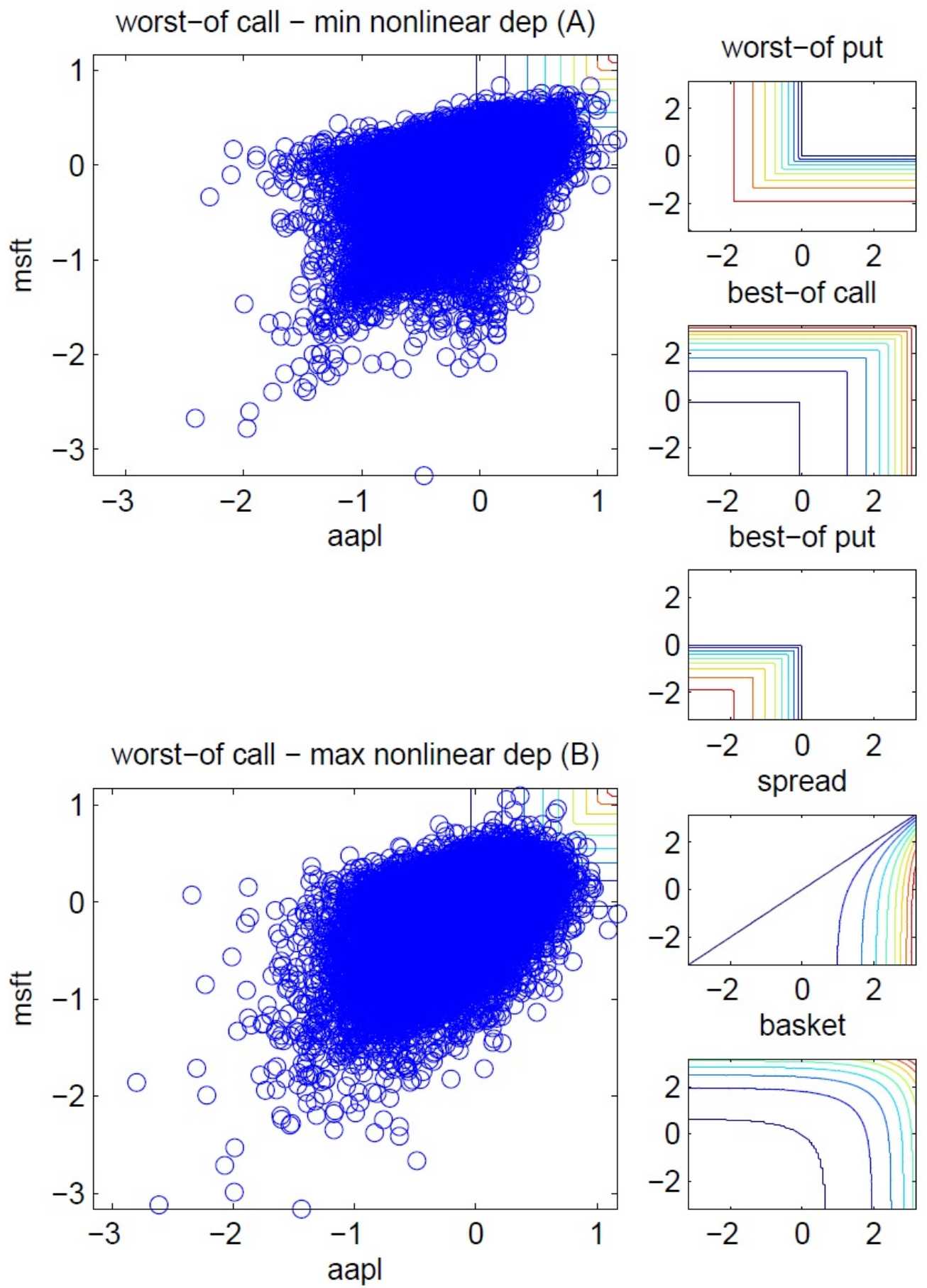

Figure 7: Scatter plots of 1 year log-returns. Apple and Microsoft, October 26, 2016 $($ SatoNIG model - correlation level $=0.5)$. 
regressions, as well as the impact of nonlinear dependence, which we are going to discuss below. We notice that in the high market volatility scenario (date 1) the explanatory power of the regression models tends to be lower, nevertheless the same considerations made for the dates 2 and 3 apply.

It is apparent from results in Table 7 that almost all coefficients are highly significant. As we observe a remarkable improvement in the explication power of the regression model for all option types and models when we add the comoment variations (increasing adj. $R^{2}$ ), we expect a relevant influence of nonlinear dependence on the pricing of the claims. The fact that option prices are significantly affected by the nonlinear dependence incorporated in a model indicates that the calibration procedure could be improved by considering not only correlation, but also a measure of nonlinear dependence. We can notice that the time to maturity effect is always the same in both specifications for Sato models, because we have seen that in Sato processes time is orthogonal to any other variable included in both regressions (comoments, variation and correlation). Moreover, looking at the sample size of each regression model, we notice that the number of products that can be valued within Sato models is always higher than that of Lévy models, coherently with the observation that in Sato specifications we can reach more often our highest correlation threshold of 0.9. Analysing regression coefficients estimates, we can observe that in some cases comoments variations have a scarce impact in economic terms on price variations, whereas in other cases they have a significant influence. For instance, we observe smaller absolute values of beta estimates in the worst-of put case (in particular with Sato models), so we do not expect any significant influence of nonlinear dependence on the pricing of the claim in this setting. On the contrary, we can observe greater absolute values of the estimates relative to coskewness in both worst-of call and best-of put cases. In the best-of put case we also observe the greatest absolute values of beta estimates relative to cokurtosis variation. These observations are somehow in line with what we observe in Figure 7, enhancing and completing the results found in the regression analysis: the differences between these two scenarios are more severe depending on the shape of the iso-price curves, so depending on the specific payoff function of the multivariate derivative under consideration. 


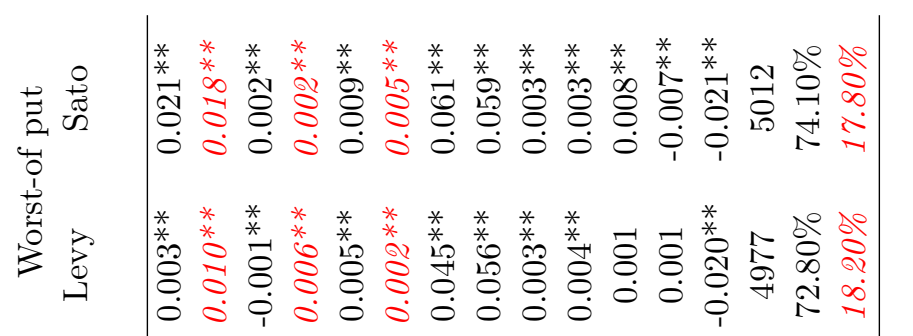

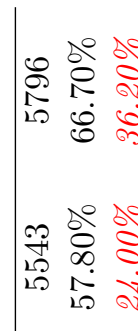

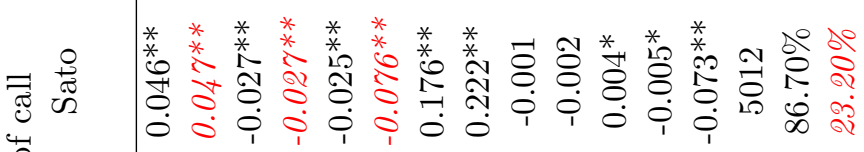

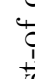

$\stackrel{3}{0}$

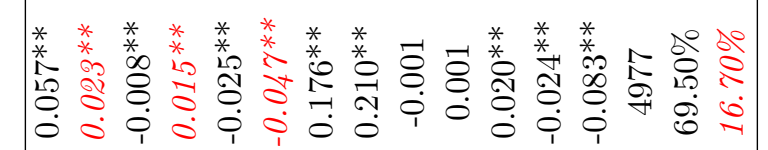

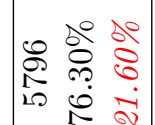

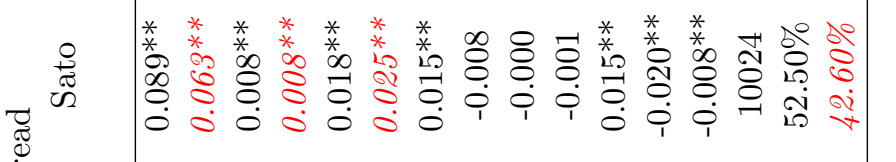

ڤ̆

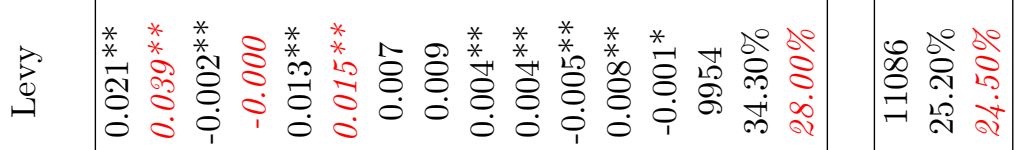

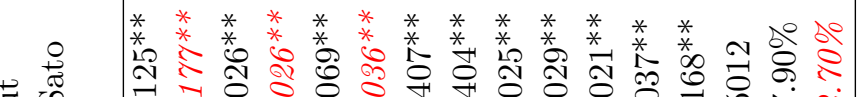

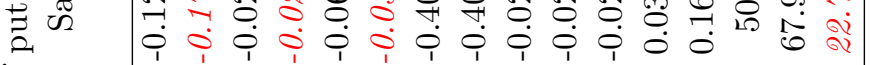

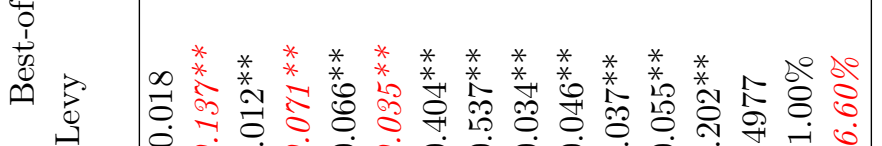

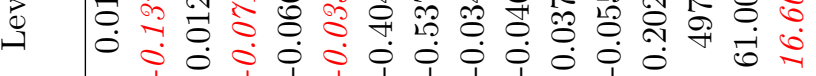

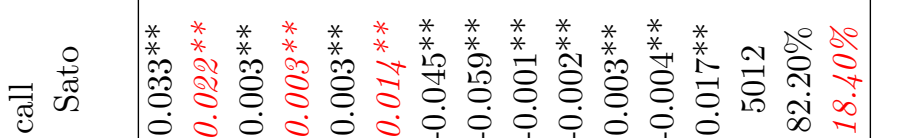

\begin{tabular}{lll}
1 & 0 & 1 \\
\hdashline & 8 & 8 \\
2 & 8 & 0
\end{tabular}

in 80 का

प्र

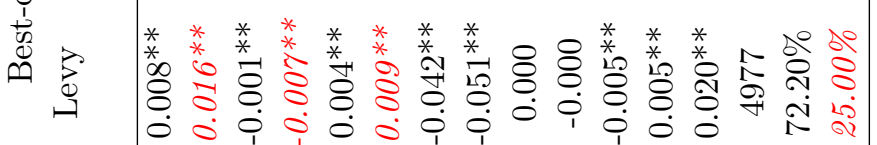

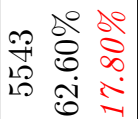

๑ั

|

$\therefore$ 造 de

年

กิ

का

ำ

जि के

\&

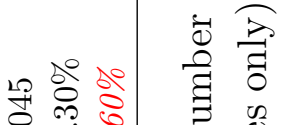

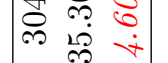

光

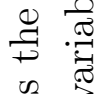

$\because$ do de

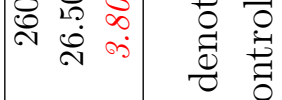

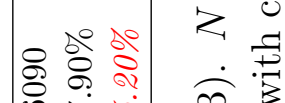

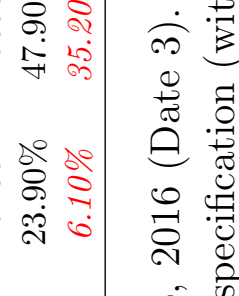

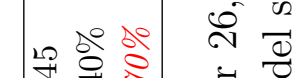

강

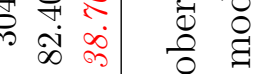

:

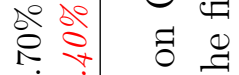

न मुं क्ष

ㅎํㅇ se

(⿸丆口

욜

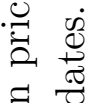

in

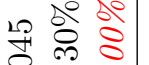

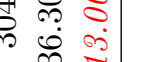

ฮี

용

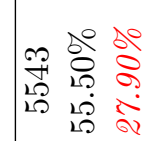

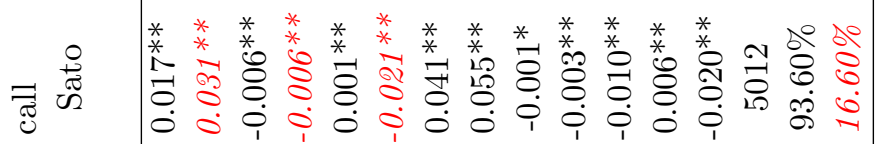

일

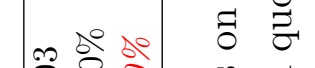

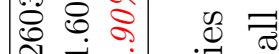

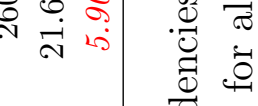

एक

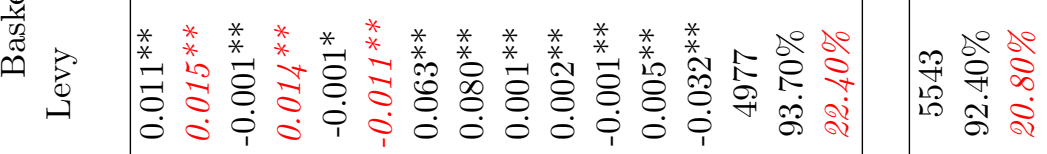

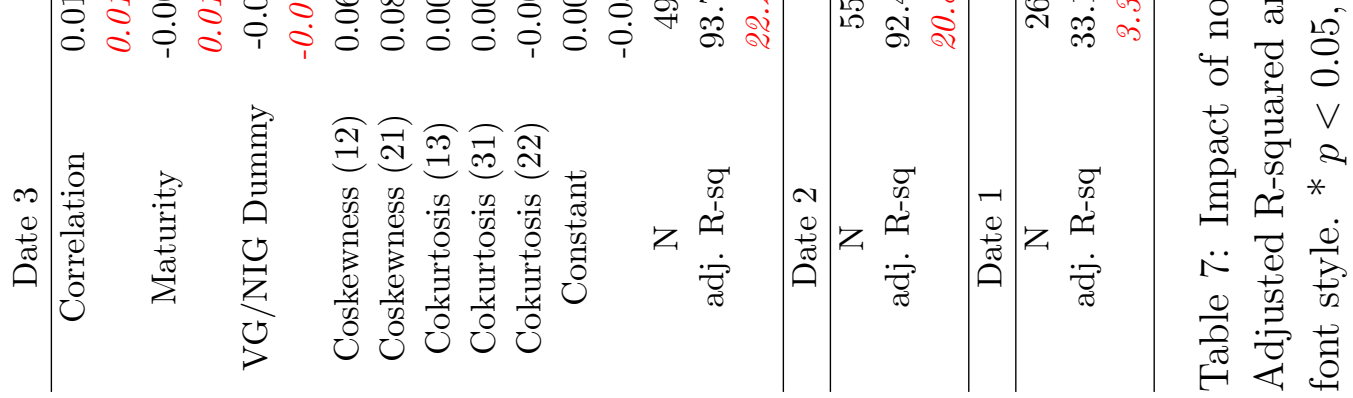




\section{Conclusion}

In the risk neutral setting, Sato models provide a significant material gain to practioners with respect to Lévy models in terms of marginal fit in both time to maturity and strike dimensions. In fact Lévy models should be calibrated to one maturity at a time or on maturity buckets to provide a reasonable fit. At the multivariate level, Guillaume proposed a model which incorporates the marginal properties of Sato processes and the dependence structure of the $\alpha$-models. Since the dependence flexibility of the $\alpha$ models has been significantly improved by the more general $\rho \alpha$-models, here we introduce a class of multivariate models with the dependence structure of $\rho \alpha$-models and Sato marginal distributions. In a multivariate framework, given the trade-off of marginaldependence for $\rho \alpha$-models, we need to assess if the correlation flexibility is preserved in the Sato setting. Comparing the performance of $\rho \alpha$-Lévy and $\rho \alpha$-Sato models it comes out that, as expected, Sato models outperform the marginal fit of plain vanilla markets in both strike and maturity dimensions. Furthermore, in our datasets, Sato marginal parameters reveal to be less binding for the correlation fit than the Lévy marginal ones in a higher percentage of baskets. Indeed, although the bounds on the parameters driving correlations are more restrictive, Sato models tend to span a higher range of achievable correlations than the Lévy counterpart. A sensitivity analysis shows that the nonlinear dependence incorporated in this class of models has a significant impact on derivative prices. Therefore, the calibration procedure could be improved by considering not only correlation, but also a measure of nonlinear dependence, especially for most sensitive products. This feature is incorporated in practice in the calibration step if multivariate option quotes are available for some specific products.

\section{Appendices}

\section{A Model correlations}

Applying Theorem 3.3 in Barndorff-Nielsen et al. (2001) and its univariate version (Theorem 30.1 in Sato (1999)) to $\boldsymbol{Y}^{I}(t)=\left(B_{1}\left(X_{1}(t)\right), \ldots, B_{1}\left(X_{1}(t)\right)\right)$ and $\boldsymbol{Y}^{\rho}(t)=$ $\left(B_{1}^{\rho}(Z(t)), \ldots, B_{n}^{\rho}(Z(t))\right)$, we find the characteristic function $\psi_{\boldsymbol{Y}(t)}$ of $\boldsymbol{Y}(t)$

$$
\begin{aligned}
\psi_{\boldsymbol{Y}(t)}(\boldsymbol{u}) & =\psi_{\boldsymbol{Y}^{I}(t)}(\boldsymbol{u}) \psi_{\boldsymbol{Y}^{\rho}(t)}(\boldsymbol{u})= \\
& =\exp \left(t \sum_{j=1}^{n} l_{X_{j}}\left(\log \left(\psi_{B_{j}}\left(u_{j}\right)\right)\right)\right) \exp \left(t l_{Z}\left(\log \left(\psi_{\boldsymbol{B}^{\rho}}(\boldsymbol{u})\right)\right)\right)
\end{aligned}
$$


where $l_{X_{j}}$ and $l_{Z}$ are the Laplace exponents of the subordinators. Correlations are independent of time and are given by

$$
\begin{aligned}
\rho_{\boldsymbol{Y}}(i, j) & =\frac{\operatorname{Cov}\left(B_{i}^{\rho}, B_{j}^{\rho}\right) E(Z)+E\left(B_{i}^{\rho}\right) E\left(B_{j}^{\rho}\right) V(Z)}{\sqrt{V\left(Y_{i}\right) V\left(Y_{j}\right)}} \\
& =\frac{\rho_{i j} \sigma_{i} \sigma_{j} \sqrt{\alpha_{i}} \sqrt{\alpha_{j}} E(Z)+\mu_{i} \mu_{j} \alpha_{i} \alpha_{j} V(Z)}{\sqrt{V\left(Y_{i}\right) V\left(Y_{j}\right)}} .
\end{aligned}
$$

A discussion on correlation features and dependence structure of these models can be found in Luciano et al. (2016) and Marena et al. (2015).

\section{B Specifications}

\section{B.1 Variance Gamma}

The VG univariate process, introduced by Madan and Seneta (1990), is a real Lévy process $L_{V G}=\left\{L_{V G}(t), t \geq 0\right\}$ which can be obtained as a Brownian motion timechanged by a Gamma process $\{G(t), t \geq 0\}$. Let $\sigma>0$ and $\mu$ be real parameters, then the process $L_{V G}$ is defined as

$$
L_{V G}(t)=\mu G(t)+\sigma B(G(t))
$$

where $B$ is a standard Brownian motion. Its characteristic function is

$$
\psi_{V G}(u)=\left(1-i u \mu \alpha+\frac{1}{2} \sigma^{2} \alpha u^{2}\right)^{-\frac{t}{\alpha}} .
$$

We now specify $\boldsymbol{G}$ to have Gamma marginal distributions. Let $X_{j}$ and $Z$ be distributed according to Gamma laws:

$$
\mathcal{L}\left(X_{j}\right)=\Gamma\left(\frac{1}{\alpha_{j}}-a, \frac{1}{\alpha_{j}}\right) \quad \text { and } \quad \mathcal{L}(Z)=\Gamma(a, 1),
$$

and let the parameters $\alpha_{j}$ and $a$ satisfy the constraints $0<\alpha_{j}<\frac{1}{a}, j=1, \ldots, n$. The subordinator $\boldsymbol{G}(t)$ has marginals $\mathcal{L}\left(G_{j}\right)=\Gamma\left(\frac{1}{\alpha_{j}}, \frac{1}{\alpha_{j}}\right)$ and the process $\boldsymbol{Y}$ defined in (1.1) has VG marginal processes with parameters $\mu_{j}, \alpha_{j}, \sigma_{j}$ - denoted as $V G\left(\mu_{j}, \alpha_{j}, \sigma_{j}\right)$ - i.e.

$$
\mathcal{L}\left(Y_{j}\right)=\mathcal{L}\left(\mu_{j} G_{j}(t)+\sigma_{j} W\left(G_{j}(t)\right)\right)
$$

We name $\boldsymbol{Y}$ a $\rho \alpha$-Variance Gamma process, shortly $\rho \alpha \mathrm{VG}$.

By imposing $\rho_{i j}=0$, for $i \neq j, \rho_{i j}=1$, for $i=j$, we find as a subcase of the current model the $\alpha$-VG process introduced in Semeraro (2008). 


\section{B.2 Normal inverse Gaussian}

A NIG process with parameters $\gamma>0,-\gamma<\beta<\gamma, \delta>0$ is a Lévy process $L_{N I G}=$ $\left\{L_{N I G}(t), t \geq 0\right\}$ with time one characteristic function

$$
\psi_{N I G}(u)=\exp \left(-\delta\left(\sqrt{\gamma^{2}-(\beta+i u)^{2}}-\sqrt{\gamma^{2}-\beta^{2}}\right)\right) .
$$

It can be constructed by subordinating a Brownian motion with an Inverse Gaussian distribution. Let

$$
X_{j} \sim I G\left(1-a \sqrt{\alpha_{j}}, \frac{1}{\sqrt{\alpha_{j}}}\right), j=1, \ldots, n \quad \text { and } \quad Z \sim I G(a, 1),
$$

where

$$
0<a<\frac{1}{\sqrt{\alpha_{j}}}, \quad j=1, \ldots, n
$$

let now $\gamma_{j}, \beta_{j}, \delta_{j}$ be such that

$$
\gamma_{j}>0, \quad-\gamma_{j}<\beta_{j}<\gamma_{j}, \quad \delta_{j}>0
$$

further, let

$$
\frac{1}{\sqrt{\alpha_{j}}}=\delta_{j} \sqrt{\gamma_{j}^{2}-\beta_{j}^{2}}
$$

If we set $\mu_{j}=\beta_{j} \delta_{j}^{2}$ and $\sigma_{j}=\delta_{j}$ in the process $\boldsymbol{Y}$ defined in (1.1), then $\boldsymbol{Y}$ has NIG marginal processes, i.e.

$$
\mathcal{L}\left(Y_{j}(t)\right)=\mathcal{L}\left(\beta_{j} \delta_{j}^{2} G_{j}(t)+\delta_{j} W\left(G_{j}(t)\right)\right)
$$

We name $\boldsymbol{Y}$ a $\rho \alpha$-Normal Inverse Gaussian process, shortly $\rho \alpha$-NIG.

\section{References}

Barndorff-Nielsen, O. E., Pedersen, J., and Sato, K. (2001). Multivariate subordination, self-decomposability and stability. Advances in Applied Probability, pages 160-187.

Boen, L. and Guillaume, F. (2016). Towards a-gamma Sato multivariate model. Available at SSRN: https://ssrn.com/abstract=2900900.

Carr, P., Geman, H., Madan, D. B., and Yor, M. (2007). Self-decomposability and option pricing. Mathematical finance, 17(1):31-57.

Eberlein, E. and Madan, D. (2010). On correlating Lévy processes. The Journal of Risk, 13:3-16.

Eberlein, E. and Madan, D. B. (2009). Sato processes and the valuation of structured products. Quantitative Finance, 9(1):27-42. 
Finlay, R. and Seneta, E. (2008). Option pricing with vg-like models. International Journal of Theoretical and Applied Finance, 11(08):943-955.

Guillaume, F. (2012). Sato two-factor models for multivariate option pricing. Journal of Computational Finance, 15(4):159.

Jackson, K., Jaimungal, S., and Surkov, V. (2007). Fourier space time-stepping for option pricing with Lévy models. Journal of Computational Finance, 12.

Luciano, E., Marena, M., and Semeraro, P. (2016). Dependence calibration and portfolio fit with factor-based subordinators. Quantitative Finance, pages 1-16.

Luciano, E. and Schoutens, W. (2006). A multivariate jump-driven financial asset model. Quantitative finance, 6(5):385-402.

Luciano, E. and Semeraro, P. (2010). Multivariate time changes for Lévy asset models: Characterization and calibration. Journal of Computational and Applied Mathematics, 233(8):1937-1953.

Madan, D. B. and Schoutens, W. (2013). Simple processes and the pricing and hedging of cliquets. Mathematical Finance, 23(1):198-216.

Madan, D. B. and Seneta, E. (1990). The variance gamma model for share market returns. Journal of business, 63:511-524.

Marena, M., Romeo, A., Semeraro, P., et al. (2015). Pricing multivariate barrier reverse convertibles with factor-based subordinators. Technical report, Collegio Carlo Alberto.

Marfè, R. (2012). A generalized variance gamma process for financial applications. Quantitative Finance, 12(1):75-87.

Sato, K.-I. (1991). Self-similar processes with independent increments. Probability Theory and Related Fields, 89(3):285-300.

Sato, K.-i. (1999). Lévy processes and infinitely divisible distributions. Cambridge University Press.

Semeraro, P. (2008). A multivariate variance gamma model for financial applications. International Journal of Theoretical and Applied Finance, 11(01):1-18.

Storn, R. and Price, K. (1997). Differential evolution - a simple and efficient heuristic for global optimization over continuous spaces. Journal of global optimization, 11(4):341359 .

Takano, K. (1989). On mixtures of the normal distribution by the generalized gamma convolutions. Bulletin of the Faculty of Science, Ibaraki University. Series A, Mathematics, 21:29-41. 
Tankov, P. (2006). Simulation and option pricing in Lévy copula models. Mathematical Modelling of Financial Derivatives, IMA Volumes in Mathematics and Applications, Springer.

Urbanik, K. (1978). Lévy's probability measures on Banach spaces. Studia Mathematica, 63(3):283-308. 

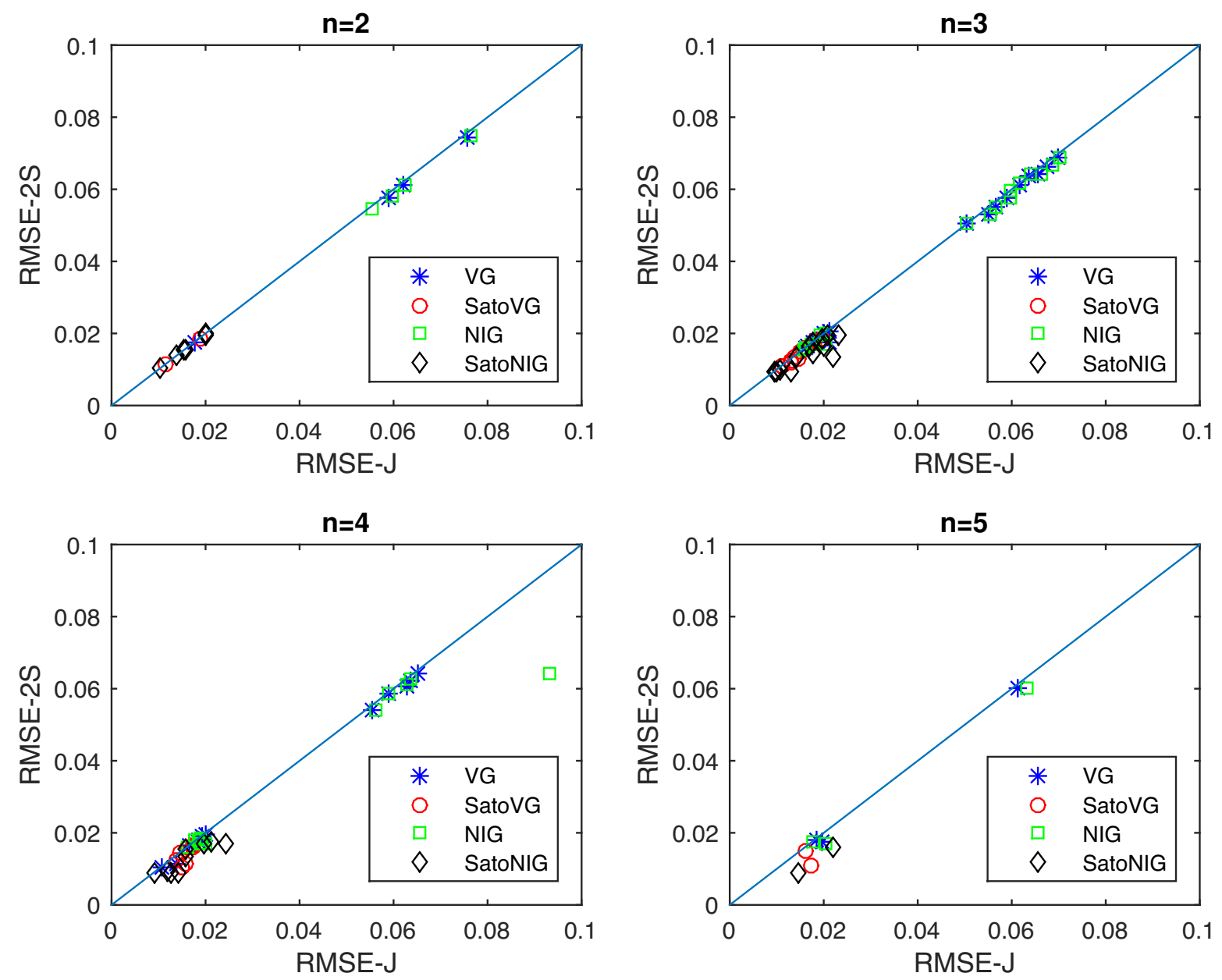

Figure 8: Average RMSE in the two-step vs the joint calibration procedure for each basket and different basket sizes $n=2,3,4,5$. Only baskets for which the two-step calibration procedure provides a correlation maximum absolute error higher than 0.1 are reported. 